\title{
Azithromycin with nitazoxanide, hydroxychloroquine or ivermectin, with or without dutasteride, for early stage COVID-19: an open-label prospective observational study in males with mild-to-moderate COVID-19 (The Pre-AndroCoV Male Trial).
}

Flavio A Cadegiani ( $\square$ flavio.cadegiani@unifesp.br)

Corpometria Institute https://orcid.org/0000-0002-2699-4344

Andy Goren

Applied Biology Inc.

Carlos G Wambier

Brown University

John McCoy

Applied Biology Inc.

Research Article

Keywords: COVID-19, SARS-CoV-2, antiandrogen, dutasteride, spironolactone

Posted Date: October 20th, 2020

DOI: https://doi.org/10.21203/rs.3.rs-88952/v2

License: (c) (i) This work is licensed under a Creative Commons Attribution 4.0 International License. Read Full License 


\section{Abstract}

Background: COVID-19 is a multisystemic disorder caused by SARS-CoV-2 that has led to more than $1,000,000$ deaths until the end of September 2020. Besides aging, obesity, and metabolic diseases, males, in particular those affected by androgenetic alopecia (AGA), are at higher risk to develop complications. While policies for diagnosis of COVID-19 still focus on the presence of fever or shortness of breath, these symptoms tend to appear only in later and more severe stages of the disease, when viral infectivity is already, hampering potential antiviral approaches. In addition, clinical characterization of early COVID-19 stages still lacks. The objective of the present observational study was to characterize prospectively clinical features and predictors in males during early COVID-19, and to evaluate whether the combination of more sensitive case-detection, early diagnosis and early pharmacological approaches would lead to improved clinical outcomes.

Material and methods: Males confirmed for COVID-19 through positive real-time polymerase chain reaction (rtPCR) for SARS-CoV-2 with less than seven days of symptoms and three days of COVID-19 confirmation were divided into non-AGA, AGA not using dutasteride (AGA no-5ARi), and AGA using dutasteride (AGA-5ARi) groups. Patients were actively characterized for baseline and lifestyle characteristics, 22 different diseases, 42 drug classes and vaccines, 26 different symptoms, and 10 different parameters to measure COVID-19 related clinical outcomes. Azithromycin plus hydroxychloroquine, nitazoxanide $500 \mathrm{mg}$ or ivermectin, with or without dutasteride or spironolactone were used. Patients were then evaluated for COVID-19 clinical course, duration, and progression.

Results: A total of 305 males were enrolled, including 192 non-AGA, 71 AGA non-ARi and 52 AGA-5ARi. The prevailing symptoms were anosmia (68.9\%), ageusia (61.2\%), headache (37.5\%), hyporexia (37.5\%), fatigue (35.2\%), dry cough (35.2\%), fever or "feverish" (33.9\%), thoracic pain (32.4\%), conjunctival hyperemia $(29.5 \%)$, weakness $(29.5 \%)$, nasal congestion or rhinorrhea ( $28.6 \%$ and myalgia $(26.3 \%)$. ARi users remained asymptomatic throughout COVID-19 treatment in $82.7 \%$ (43 of 52 males), and the only symptoms present in more than two AGA-5ARi patients were anosmia and ageusia. Thoracic, upper back, lower back pain, arthralgia affected a higher percentage of AGA no-5ARi than non-AGA males (all $p<$ $0.01)$, but had similar durations ( $p=n / s)$. Anosmia, ageusia, headache, fatigue, myalgia and conjunctival hyperemia were more commonly present and lasted for longer periods in AGA no 5ARi patients (all $p<$ 0.01). Self-reported perception of "sinusitis" and "sore throat", dry cough and weakness were equally present $(p=n / s)$ but had longer duration in AGA no-5ARi males (all $p<0.01)$.

The different drug combinations were equally distributed $(p>0.05)$. AGA males were more severely affected than non-AGA in terms of duration of clinical manifestations ( $9.4 \pm 6.0$ vs $14.2 \pm 7.3$ days, $p<$ $0.0001)$ and viral shedding ( $14.0 \pm 5.2$ vs $17.8 \pm 6.2$ days; $p<0.0001)$, which has been fully mitigated by the chronic use of dutasteride ( $p<0.0001$ and $<0.0001$ vs non-AGA and AGA no-5ARi, respectively, for both clinical manifestations and viral shedding duration. Non-AGA, AGA no-5ARi and AGA-5ARi achieved $95 \%$ clinical recovery in seven, 14 , and two days, respectively. In regards functional capacity, AGA no -5ARi males at Days 30,14, 7, and 3 after treatment initiation were similar than non-AGA at Days $14,7,3$, and 0 , 
respectively (all $p>0.9$ ). None of the patients required hospitalization and mechanical ventilation, or progressed to more severe states.

Conclusion: The combination of more sensitive and earlier diagnosis of COVID-19 with a variety of drug combinations with preliminary demonstration of direct or indirect antiviral activity against SARS-CoV-2 demonstrated indisputable improved COVID-19 related clinical outcomes compared to the extensively described COVID-19 clinical course, and avoided the progression to more severe state in all patients included in the present analysis, independently of risk factors, demonstrating that any additional risk factor can be completely mitigated by the combination of more sensitive clinical suspect with early pharmacological approaches.

The overwhelming differences indicate that full placebo control RCTs for early COVID-19 may be ethically questionable. Instead, double blind therapies with different options, or mixed open label placebo control for COVID-19 should be considered.

\section{Background}

COVID-19 is a multi-systemic infection caused by the Severe Acute Respiratory Syndrome Coronavirus 2 (SARS-CoV-2) that has infected more than $6,000,000$ people and killed more than $1,000,000$ people worldwide until the end of September 2020. Aging, obesity, uncompensated type 2 diabetes mellitus (T2DM), hypertension, male sex, and presence of male androgenetic alopecia (AGA) have been detected as risk factors for COVID-19 complications (1-6).

Although mortality in COVID-19 is slightly decreasing along the pandemics, challenges for early detection and management remain, and include the large number of asymptomatic yet transmitting subjects, prolonged viral incubation and shedding period, lack of specific and highly heterogeneous clinical manifestations (except for anosmia and ageusia), and rapid progression to acute lung injury from oligosymptomatic clinical presentations (7-10).

Despite the challenging detection of suspected cases during the earlier stages, the majority of the public policies still focus on the presence of fever or shortness of breath as sine-quo-non criteria for the performance of real-time Polymerase Chain Reaction (rtPCR) (11). This hampers the diagnosis of COVID19 without the presence of any of these two symptoms, and keeps medical literature claims on the presence of fever in the vast majority of infected patients, despite more specific and independent extensive data showing otherwise $(12,13)$.

The natural course of SARS-CoV-2 has been divided into three major stages: the first one encompasses the period of SARS-CoV-2 viral infectivity and spread; the second stage corresponds to an exacerbated, diffuse and dysfunctional inflammatory reaction to SARS-CoV-2, while the third stage represents the COVID-19 induced acute lung injury per se (14). 
Multiple drugs have been proposed to improve COVID-19 clinical outcomes, from the demonstration of specific in vitro antiviral activity against SARS-CoV-2, through clinical observations, or following mechanistical rationale (14). Among these drugs, azithromycin, in association with hydroxychloroquine or ivermectin, have been the most attempted drug combinations to decrease COVID-19 severity and complications (15).

However, mandatory presence of fever in order to suspect for COVID-19 prevents its detection during the first stage, when antiviral approaches should work. Indeed, since detection of COVID-19 is still relatively delayed in the majority of the cases, antiviral approaches tend to become less effective. Consequently, the vast majority of randomized clinical trials (RCT) for COVID-19, have been performed in hospitalized patients, even those claiming to be performed in mild-to-moderate COVID-19. Naturally, antiviral the alleged early pharmacological approaches for COVID-19 remain controversial, since actual early or mild presentations of COVID-19 have been under-investigated, which precludes from conclusive findings regarding the efficacy of these approaches.

For the evaluation of potential antiviral therapies of the proposed drugs may act reducing viral infectivity, it is critical to detect COVID-19 during earlier stages, based on a more sensitive case-detection guidance.

We hypothesized that the missing gap to respond to the efficacy of early approaches against COVID-19 in the current lack of proven options to prevent its progression to more severe stages is to provide potential antiviral therapies during the first stage of COVID-19, which requires more sensitive diagnosis of COVID19. Owing to the extreme heterogeneity of the early COVID-19 clinical manifestations and to the fact that because of the spread use of masks, upper respiratory tract infection (URTI) and other infections became less likely than COVID-19, we moved from the need of fever or shortness of breath to the occurrence of absolutely any symptom as suspected case of COVID-19.

The present study is a prospective observational study aiming to evaluate whether more clinical sensitive detection of COVID-19 associated with early use of the unproven yet plausible drug combinations would be able to change the course of COVID-19 by improving clinical outcomes. The present prospective observational study also aimed to drive the design of our currently ongoing RCT (ClinicalTrials.gov Identifier: NCT04446429. Available at clinicaltrials.gov (https://clinicaltrials.gov/ct2/show/NCT04446429?term=NCT04446429\&draw=2\&rank=1).

Due to distinct characteristics and disease patterns in COVID-19, particularly because of the critical function of the transmembrane serine protease 2 (TMPRSS-2), an androgen-driven protein, for the modulation of the SARS-CoV-2 cell entry and COVID-19 severity, the present prospective observational study was divided in to males and females and in the presence or absence of androgenetic alopecia (AGA) in males, since we hypothesized that responses could be sex-specific, which could provide potential insights for individualization of COVID-19 pharmacological approaches.

\section{Materials And Methods}




\section{Subject selection}

Patients were recruited both direct- and indirectly through social media and different emergency units, outpatient clinics and medical private practice centers, respectively, located in Brasilia, Brazil, in case of suspected or confirmed COVID-19.

Male candidates were suspected for COVID-19 in case of presence of at least one symptom encompassing the respiratory, gastrointestinal, neurological, cardiovascular systems, or any unspecific symptom, not limited to fever, shortness of breath, anosmia or ageusia. Suspected cases underwent rtPCR-SARS-CoV-2 (Abbott RealTime SARS-CoV-2 Assay, Abbott, USA; or Cobas SARS-CoV-2, Roche, Switzerland), and those confirmed for SARS-CoV-2 were included in the study if inclusion criteria were met. Patients confirmed for COVID-19 and referred by external medical centers were selected if they met criteria for the present study.

For patients with confirmed COVID-19 through positive rtPCR-SARS-CoV-2, inclusion criteria for the present study included: 1 . Male sex; 2.18 years old and above; 3 . Less than seven days since the beginning of symptoms; 4 . Less than 72 hours from the confirmation of COVID-19; 4 . Lack of use of any drug with potential antiviral activity against SARS-CoV-2, including hydroxychloroquine, nitazoxanide and ivermectin, and glucocorticoids, prior to the study; and 5. Absence of clinical or radiological evidence of progression to second or third stage of COVID-19, severe lung injury, or severe COVID-19. Criteria for exclusion including shortness of breath, oxygen saturation (SatO2) lower than $92 \%$, and more than $25 \%$ of lungs affected in a chest computed tomography (CT) scan.

Patients included for the present analysis provided a written consent exactly as approved by the Institutional Review Board (IRB) of the Ethics Committee of the National Board of Ethics Committee of the Ministry of Health, Brazil (CEP/CONEP: Parecer 4.173.074 / CAAE: 34110420.2.0000.0008), alongside with the currently ongoing randomized clinical trial (RCT) registered at ClinicalTrials.gov (Identifier: NCT04446429. Available at clinicaltrials.gov (https://clinicaltrials.gov/ct2/show/NCT04446429? term=NCT04446429\&draw=2\&rank=1).

\section{Design and methods}

This is a prospective observational study of patients confirmed for COVID-19 after suspected for COVID19 through a sensitive case-detection basis. For this, a preliminary 'pre-study' phase was conducted with males that presented any symptom, not restricted to those observed in URTIs, anosmia or ageusia, as being suspected for COVID-19, that yielded a positive rtPCR-SARS-CoV-2 (Abbott RealTime SARS-CoV-2 Assay, Abbott, USA; or Cobas SARS-CoV-2, Roche, Switzerland). Not only new onset symptoms, but also changes in patterns of chronic symptoms, like headache or cough, were also considered as suspected for COVID-19. No history of contact with confirmed case of COVID-19 was required.

Included males were actively characterized for baseline and lifestyle characteristics, 22 different diseases, 42 drug classes and vaccines, 26 different symptoms, and 10 different parameters to measure COVID-19 
related clinical outcomes. All parameters that were actively characterized are listed in Table 1. Males were then divided according to the presence or absence of androgenetic alopecia (AGA), and use or not of dutasteride. Male AGA was considered when front or scalp hair was decreased in a male loss pattern, controlled with specific drugs (finasteride or dutasteride), or previous hair transplant. For statistical and clinical purposes, severe hyposmia and dysgeusia were considered as anosmia and ageusia, respectively.

Further, clustering of clinical manifestations was performed due to the overwhelming grouping of symptoms. The following clusters have been identified: 1 . Anosmia-Ageusia dominance; 2. Dengue-like symptomatology / clinical presentation; 3. Upper respiratory tract infection (URTI) URTI-like symptomatology / clinical presentation; 4. Gastrointestinal (GI) infection-like symptomatology / clinical presentation; 5. Mixed between clusters; 6 . Unspecific presentation; or 7. Asymptomatic, as shown in Figure 1.

To fill criteria for each cluster, it has been required for:

1. Anosmia-Ageusia dominance: at least two of nasal congestion or rhinorrhea, dry cough, self-reported perception of "sinusitis", or self-reported perception of "sore throat";

2. Dengue-like clinical presentation: at least three of myalgia, arthralgia, upper back pain, conjunctival hyperemia or pre-orbital pain;

3. URTI-like clinical presentation: at least two of nasal congestion or rhinorrhea, dry cough, self-reported perception of "sinusitis", or self-reported perception of "sore throat";

4. GI infection-like clinical presentation: at least two of diarrhea, nauseas, vomiting, or abdominal pain;

5. Mixed between clusters: when there are symptoms to fill criteria for at least two clusters

6. Unspecific presentation: when there are only unspecific or insufficient symptoms to fill criteria for any cluster; or

7. Asymptomatic.

After characterization, azithromycin was given at a dose of $500 \mathrm{mg}$ per day for five days, in association with hydroxychloroquine at a daily dose of $400 \mathrm{mg}$ for five days, nitazoxanide $500 \mathrm{mg}$ bis in die for six days, or ivermectin $0.2 \mathrm{mg} / \mathrm{kg} /$ day in a single daily dose for three days. Optionally, dutasteride, spironolactone, vitamin D, vitamin C, zinc, apibaxan, rivaroxaban, enoxaparin, and glucocorticoids were prescribed. The choice between hydroxychloroquine, nitazoxanide, and/or ivermectin, as well as the optional use dutasteride, spironolactone (if applicable) and any other drug or supplement was based on clinical judgement, availability, and individual medical history.

Patients were then evaluated for disease course and progression, time-to-appearance and duration of each symptom, and severity scales, including hospitalization, intensive care, mechanical ventilation, and death, duration of symptoms including and also not including anosmia and ageusia, and duration of 
positive rtPCR-SARS-CoV-2. In individually selected cases, chest CT scan was performed. Full raw data is available at a data repository (https://osf.io/cm4f8/).

\section{Data availability}

Full raw data is available at a public repository, in the following electronic address: https://osf.io/cm4f8/, that has been made public available.

Sample size

Sample size was determined based on the following assumptions (22):

a. Sample size estimate for the chi-squared test will require $80 \%$ power to detect the difference in proportions at $\mathrm{p}=0.05$

b. $95 \%$ of subjects would complete the study

c. Hospitalization and death rates between 3 and $20 \%$, and 0.3 and $2.5 \%$, respectively $(1,2,6,23)$

Based on the assumptions above, we calculated that at a minimum we would need to recruit 254 subjects, and the study terminated when this number was reached, or in case of unexpected outcomes that could justify its early termination.

Statistical analysis

Nonparametric ANOVA (Kruskal-Wallis) was performed for all parameters, regardless of the distribution normality, and post-hoc adjusted Dunn's test was performed for subgroup analyses, whenever $p<0.05$. All statistical tests were performed using XLSTAT version 22.4.1 (Microsoft, USA).

Table 1. Parameters evaluated for the present prospective observational study. 
Parameter

\section{Baseline characteristics}

Age $(y / 0)$

Weight $(\mathrm{kg})$

Height (m)

$\operatorname{BMI}\left(\mathrm{kg} / \mathrm{m}^{2}\right)$

Married (yes/no) and households (yes/no)

\section{Disease and treatment timing}

Time-to-treat (interval between beginning of symptoms and beginning of specific treatment) (days)

Duration of positive rtPCR SARS-CoV-2 (days)

Duration of symptoms (not including anosmia and ageusia) (days)

Duration of symptoms (including anosmia and ageusia) (days)

\section{Medical history}

Existing diseases

Hypertension

Myocardial infarction

Stroke

Chronic heart failure

Lipid disorders

Type 2 diabetes mellitus (T2DM)

Pre-diabetes

Dysglicaemia (T2DM + pre-diabetes)

Obesity

Asthma

Chronic Obstructive Pulmonary Disease (COPD)

Chronic Kidney Disease (CKD)

Liver fibrosis or cirrhosis

Major depression

Anxiety or anxiety-related disorders 
Attention deficiency and hyperactive disorders (ADHD)

Insomnia

Hypothyroidism

Autoimmune disorders (any)

Current or previous cancer

Hypogonadism

Benign Prostate Hyperplasia (BPH)

Other diseases (any)

\section{Current medications}

Cardiovascular

drugs
Beta-blocker

Angiotensin converter inhibitors (ACEi) (-pril)

Angiotensin-2 receptor blockers (ARB) (-tan)

Loop diuretics (furosemide)

Thiazide diuretics (hydrochlorotiazide (HCTZ), indapamide)

Calcium channel blockers (CCB) (-dipine)

K-sparing diuretics (spironolactone)

Statins (pitava-, rosuva-, atorva-, prava-, simvastatin)

Other lipid-lowering agents (fibrates, ezetimibe, PCSK9 inhibitors)

Aspirin

Clopidogrel

Warfarin

Xa factor inhibitors (apibaxan, rivaroxaban)

Direct thrombin inhibitors (dabagatran)

Heparins
Diabetes, obesity, and metabolicrelated drugs
Biguanides (metformin)

Glucagon-like peptide 1 (GLP1) receptor analogues (GLP1Ra) (lira-, sema-, dulaglutide; exenatide)

Sodium-glucose co-transporter 2 (SGLT2) inhibitors (SGLT2i) (empa-, dapa-, canagliflozin) 
Di-peptyl peptidase 4 (DPP4) inhibitors (DPP4i) (vilda-, sita-, saxa-, linagliptin)

Sulfonylureas (glipizide, glimepiride, glicazide)

Glitazone

Acarbose

Insulin

Orlistat

Hormone and related therapies

Hormone and related therapies
Levothyroxine (with or without liothyronine)

Testosterone (any regimen)

Aromatase inhibitors (anastrozole; letrozole)

Selective estrogen receptor modulators (SERMs)

5-alpha reductase inhibitors (dutasteride, finasteride)

Gonadotropin releasing hormone $(\mathrm{GnRH})$ analogues and inhibitors

Non-steroidal antiandrogens (NSAA)

Central-acting drugs

Other drugs

Hypnotics (zolpidem, zopiclone, eszopiclone, ramelteon)

Selective serotonin reuptaker inhibitors (SSRIs) (fluoxetine, (des)venlafaxine, sertraline, (es)citalopram, vortioxetine, fluvoxamine)

Other antidepressants and humor stabilizers (bupropion, topiramate, trazodone, ami- ou nortriptiline, topiramate, oxcarbamazepine)

Benzodiazepines (Lora-, broma-, dia-, clonazepam; alpra-, midazolam)

Atypical antipsychotics (olanzapine, quetiapine, risperidone, clozapine, aripiprazole)

Central nervous system (CNS) stimulants (methylfenidate, lisdexamfetamine, modafinil)

Alpha-1 adrenergic blockers (prazosin, doxazosin, tamsulosin)

Phosphodiesterase 5 inhibitors (sildenafil, tadalafil)

Supplements

Omega-3 (> 3g/day)

Vitamina $D(>1,000$ iu/day $)$

Zinc (> 15mg/day)

Vitamin C (> 500mg/day) 
Vaccine

BCG (lifetime)

Influenza (in 2020)

Pneumococcal 13 or 23 (since 2017)

\section{Lifestyle}

Current smoking ( $>2$ packs/week and $>10$ pack-year)

Regular physical activity (>150min/week, moderate-tovigorous, > 3 METs, for > 1y)

Clinical characterization

Presence (yes/no)

Time to appearance (days)

Duration (days)

Unspecific symptoms

Fever

"Feverish"

Headache

Shortness of breath

Anosmia

Ageusia / hypergeusia / dysgeusia

Dizziness

Weakness

Fatigue

Hyporexia / anorexia

Thoracic pain

Lower back pain

Dry eyes / dry mouth / skin lesions

Testicular pain

Upper respiratory tract infectionlike symptoms
Nasal congestion or rhinorrhea

Dry cough

"Sinusitis" (self-reported perception)

"Sore throat" (self-reported perception) 
Arthralgia

Upper back pain

Conjuctival hyperemia

Pre-orbital pain

Gastrointestinal (GI) infection-like symptoms

\section{Diarrhea}

Nauseas

Vomiting

Abdominal pain

Anosmia-Ageusia dominance; or

Dengue-like symptomatology / clinical presentation; or

URTI-like symptomatology / clinical presentation; or

GI infection-like symptomatology / clinical presentation; or

Mixed; or

Unspecific; or

Asymptomatic

\section{Treatment}

(Azithromycin 500mg/day for 05 days +$)$
Hydroxychloroquine $400 \mathrm{mg} /$ day for 05 days, or

Nitazoxanide 500mg BID for 06 days, or

Ivermectin $0.2 \mathrm{mg} / \mathrm{kg} /$ day for 03 days

$+/-$

Spironolactone 100mg BID for 15 days, or

Dutasteride $0.5 \mathrm{mg} /$ day until cure

Xa factor inhibitors

Warfarin

Enoxaparin

Acetylsalicylic acid (ASA)

Glucocorticoids (methylprednisolone, dexamethasone, prednisone, prednisolone)

Bromhexine

$\mathrm{N}$-acetylcysteine 
Colchicine

Vitamin C (additional dose, if already under use)

Zinc (additional dose, if already under use)

Vitamin D (additional dose, if already under use)

Outcomes (Day $0=$ beginning of treatment)

WHO COVID Ordinal Outcomes

(Stages 1-5)

Day 0

Day 7

Day 14

Day 30

Day 60

Loss of ability to everyday activities $(0-100 ; 0=$ no loss; $100=$ complete inability)
Day 0

Day 3

Day 7

Day 14

Day 30

Symptom severity

Day -7 to -4

$(0-100 ; 0=$ worst day of symptoms; $100=$ no symptoms or fully recovered)

Day -3 to -1

Day 0
Day 1

Day 2

Day 3

Day 7

Day 14

Day 21

Day 30

Day 60

Thoracic CT scan

Day 0

(\% of lungs affected)
Day 7

Day 14

Day 30 
Brescia COVID-19 Respiratory Severity Scale (0-4)

Hospitalization

Intensive Care Unit (ICU)

Mechanical ventilation

Noradrenaline/dopamine

Death

\section{Results}

All tables depict characteristics and parameters according to the group and for overall males, as well as overall and pairwise $p$-value comparisons. Tables 2 to 5 characterize patients' background, Tables 6 to 10 describe COVID-19 presentation and proposed therapeutical approaches, and Tables 10 to 15 depict COVID-19 related outcomes.

\section{Patients' characterization}

A total of 305 males were included in the present study. Of these, 192 did not present AGA (non-AGA group) and 123 had current or history of AGA, from which 71 did not use dutasteride (AGA no-5ARi group) and 52 did (AGA-5ARi group). There was no dropout for clinical and disease progression outcomes.

Table 2 details baseline characteristics, Table 3 describes the prevalence of the most common comorbidities. Table 4 depicts the medications used on a chronic and regular basis, and Table 5 depicts vaccines and lifestyle.

Baseline characteristics (Table 2) were similar between groups, including age, body mass index (BMI), percentage of married males and percentage of males living alone, whereas non-AGA males were slightly but significantly taller than AGA males from both 5ARi and no-5ARi groups.

The major and prevailing diseases were present in similarly present in all groups (Table 3), while chronic kidney disease (CKD), liver fibrosis and cirrhosis, and current cancer were absent. Autoimmune disorders and previous cancer were present in less than three patients. None of the medications for hypertension, cardiovascular diseases, diabetes, obesity, hormonal dysfunctions and psychiatric disorders disclosed differences between groups (Table 4). Warfarin, direct thrombin inhibitors, heparins, acarbose, gonadotropin releasing hormone $(\mathrm{GnRH})$ analogues and inhibitors, non-steroidal antiandrogens (NSAA), finasteride and oral minoxidil was not used by any participant. The percentage of males that received vaccines for BCG, influenza and pneumococcal was, and that practiced physical activity regularly was similar between groups (Table 5). 
Table 2. Baseline characteristics.

\begin{tabular}{|c|c|c|c|c|c|}
\hline $\begin{array}{l}\text { Baseline } \\
\text { characteristics }\end{array}$ & $\begin{array}{l}\text { Overall } \\
\text { males } \\
(n=305)\end{array}$ & $\begin{array}{l}\text { Non-AGA } \\
\text { males } \\
(n=192)\end{array}$ & $\begin{array}{l}\text { AGA no-5ARi } \\
\text { males } \\
(n=71)\end{array}$ & $\begin{array}{l}\text { AGA-5ARi } \\
(n=52)\end{array}$ & $\begin{array}{l}\text { p-value } \\
\text { (overall) }\end{array}$ \\
\hline Age (y/o) & $43.9 \pm 10.8$ & $43.1 \pm 11.2$ & $45.2 \pm 9.0$ & $\begin{array}{l}45.5 \pm \\
11.3\end{array}$ & $\begin{array}{l}0.26 \\
(\mathrm{n} / \mathrm{s})\end{array}$ \\
\hline Height (m) & $1.76 \pm 0.06$ & $1.77 \pm 0.06^{\star \#}$ & $1.74 \pm 0.05$ & $\begin{array}{l}1.74 \pm \\
0.04\end{array}$ & $<0.0001$ \\
\hline BMI $\left(\mathrm{kg} / \mathrm{m}^{2}\right)$ & $26.9 \pm 3.7$ & $26.9 \pm 3.7$ & $26.9 \pm 4.2$ & $26.3 \pm 3.0$ & $\begin{array}{l}0.18 \\
(n / s)\end{array}$ \\
\hline Married (yes/no) & $235(76.2 \%)$ & $147(76.6 \%)$ & $52(80.3 \%)$ & $\begin{array}{l}36 \\
(69.2 \%)\end{array}$ & $\mathrm{n} / \mathrm{a}$ \\
\hline Households (yes/no) & $51(16.2 \%)$ & $29(15.1 \%)$ & $10(14.1 \%)$ & $\begin{array}{l}12 \\
(23.1 \%)\end{array}$ & $\mathrm{n} / \mathrm{a}$ \\
\hline
\end{tabular}

$\mathrm{AGA}=$ androgenetic alopecia; $5 \mathrm{ARi}=$ Androgen receptor inhibitors; $\mathrm{n} / \mathrm{s}=$ non-significant; $\mathrm{n} / \mathrm{a}=$ nonapplicable

${ }^{*} p<0.05$ vs AGA no-ARi males

${ }^{\#} \mathrm{p}<0.05$ vs AGA-ARi males

Table 3. Existing disorders. 


\begin{tabular}{|c|c|c|c|c|c|}
\hline Comorbidities & $\begin{array}{l}\text { Overall } \\
\text { males } \\
(n= \\
305)\end{array}$ & $\begin{array}{l}\text { Non-AGA } \\
\text { males } \\
(n=192)\end{array}$ & $\begin{array}{l}\text { AGA no- } \\
\text { 5ARi males } \\
(n=71)\end{array}$ & $\begin{array}{l}\text { AGA- } \\
\text { 5ARi } \\
(n=52)\end{array}$ & $\begin{array}{l}\text { p-value } \\
\text { (overall) }\end{array}$ \\
\hline Hypertension & $\begin{array}{l}39 \\
(20.3 \%)\end{array}$ & $\begin{array}{l}39 \\
(20.3 \%)\end{array}$ & $15(21.1 \%)$ & $\begin{array}{l}13 \\
(25.0 \%)\end{array}$ & $\begin{array}{l}0.74 \\
(\mathrm{n} / \mathrm{s})\end{array}$ \\
\hline Myocardial infarction & $\begin{array}{l}13 \\
(4.1 \%)\end{array}$ & $6(3.1 \%)$ & $5(7.0 \%)$ & $2(3.8 \%)$ & $\begin{array}{l}0.63 \\
(n / s)\end{array}$ \\
\hline Stroke & $4(1.3 \%)$ & $3(1.6 \%)$ & $1(1.4 \%)$ & 0 & $\mathrm{n} / \mathrm{a}$ \\
\hline Chronic Heart Failure (CHD) & $1(0.3 \%)$ & $1(0.5 \%)$ & 0 & 0 & $\mathrm{n} / \mathrm{a}$ \\
\hline Lipid disorders & $\begin{array}{l}110 \\
(34.9 \%)\end{array}$ & $\begin{array}{l}63 \\
(32.8 \%)\end{array}$ & $21(29.6 \%)$ & $\begin{array}{l}26 \\
(50.0 \%)^{\&}\end{array}$ & $\begin{array}{l}0.064 \\
(\mathrm{n} / \mathrm{s})\end{array}$ \\
\hline $\begin{array}{l}\text { Type } 2 \text { diabetes mellitus } \\
\text { (T2DM) }\end{array}$ & $\begin{array}{l}39 \\
(12.4 \%)\end{array}$ & $\begin{array}{l}21 \\
(10.9 \%)\end{array}$ & $9(21.7 \%)$ & $\begin{array}{l}9 \\
(17.3 \%)\end{array}$ & $\begin{array}{l}0.44 \\
(n / s)\end{array}$ \\
\hline Pre-diabetes & $\begin{array}{l}14 \\
(4.4 \%)\end{array}$ & $9(4.7 \%)$ & $2(2.8 \%)$ & $3(5.8 \%)$ & $\begin{array}{l}0.97 \\
(\mathrm{n} / \mathrm{s})\end{array}$ \\
\hline $\begin{array}{l}\text { Dysglycemia } \\
\text { (T2DM + pre-diabetes) }\end{array}$ & $\begin{array}{l}53 \\
(16.8 \%)\end{array}$ & $\begin{array}{l}30 \\
(15.6 \%)\end{array}$ & $11(24.5 \%)$ & $\begin{array}{l}12 \\
(24.1 \%)\end{array}$ & $\begin{array}{l}0.69 \\
(\mathrm{n} / \mathrm{s})\end{array}$ \\
\hline Obesity & $\begin{array}{l}52 \\
(16.5 \%)\end{array}$ & $\begin{array}{l}31 \\
(16.1 \%)\end{array}$ & $12(16.9 \%)$ & $\begin{array}{l}9 \\
(17.3 \%)\end{array}$ & $\begin{array}{l}0.96 \\
(\mathrm{n} / \mathrm{s})\end{array}$ \\
\hline Asthma & $\begin{array}{l}22 \\
(7.0 \%)\end{array}$ & $12(6.2 \%)$ & $8(11.3 \%)$ & $2(3.8 \%)$ & $\begin{array}{l}0.75 \\
(\mathrm{n} / \mathrm{s})\end{array}$ \\
\hline Major depression & $\begin{array}{l}12 \\
(3.8 \%)\end{array}$ & $5(2.6 \%)$ & $2(2.8 \%)$ & $5(9.6 \%)$ & $\begin{array}{l}0.54 \\
(\mathrm{n} / \mathrm{s})\end{array}$ \\
\hline Anxiety-related disorders & $\begin{array}{l}40 \\
(12.7 \%)\end{array}$ & $\begin{array}{l}22 \\
(11.5 \%)\end{array}$ & $10(14.1 \%)$ & $\begin{array}{l}8 \\
(15.4 \%)\end{array}$ & $\begin{array}{l}0.85 \\
(\mathrm{n} / \mathrm{s})\end{array}$ \\
\hline $\begin{array}{l}\text { Attention deficiency and hyperactive } \\
\text { disorders (ADHD) }\end{array}$ & $\begin{array}{l}33 \\
(10.5 \%)\end{array}$ & $18(9.4 \%)$ & $9(12.7 \%)$ & $\begin{array}{l}6 \\
(11.5 \%)\end{array}$ & $\begin{array}{l}0.69 \\
(\mathrm{n} / \mathrm{s})\end{array}$ \\
\hline Insomnia & $\begin{array}{l}25 \\
(7.9 \%)\end{array}$ & $13(6.8 \%)$ & $7(9.8 \%)$ & $5(9.6 \%)$ & $\begin{array}{l}0.61 \\
(\mathrm{n} / \mathrm{s})\end{array}$ \\
\hline Hypothyroidism & $\begin{array}{l}13 \\
(4.1 \%)\end{array}$ & $9(4.7 \%)$ & $2(2.8 \%)$ & $2(3.8 \%)$ & $\begin{array}{l}0.88 \\
(n / s)\end{array}$ \\
\hline Hypogonadism & $\begin{array}{l}34 \\
(10.8 \%)\end{array}$ & $\begin{array}{l}24 \\
(12.5 \%)\end{array}$ & $7(9.9 \%)$ & $3(5.8 \%)$ & $\begin{array}{l}0.44 \\
(\mathrm{n} / \mathrm{s})\end{array}$ \\
\hline Benign Prostate Hyperplasia (BPH) & $\begin{array}{l}13 \\
(4.1 \%)\end{array}$ & $6(3.1 \%)$ & $2(2.8 \%)$ & $5(9.6 \%)$ & $\begin{array}{l}0.18 \\
(\mathrm{n} / \mathrm{s})\end{array}$ \\
\hline
\end{tabular}


$\mathrm{AGA}=$ androgenetic alopecia; $5 \mathrm{ARi}=$ Androgen receptor inhibitors; $\mathrm{n} / \mathrm{s}=$ non-significant; $\mathrm{n} / \mathrm{a}=$ nonapplicable

${ }^{*} p<0.0001$ vs AGA no-ARi males

${ }^{\#} \mathrm{p}<0.0001$ vs AGA-ARi males

$\&_{p}<0.05$ vs no-5ARi users (non-AGA and AGA no-5ARi)

Table 4. Medications used at regular and chronic basis by COVID-19 males. 


\begin{tabular}{|c|c|c|c|c|c|}
\hline Current medications & $\begin{array}{l}\text { Overall } \\
\text { males } \\
(n= \\
305)\end{array}$ & $\begin{array}{l}\text { Non- } \\
\text { AGA } \\
\text { males } \\
(n= \\
192)\end{array}$ & $\begin{array}{l}\text { AGA no- } \\
5 A R i \\
\text { males } \\
(n=71)\end{array}$ & $\begin{array}{l}\text { AGA- } \\
5 A R i \\
(n= \\
52)\end{array}$ & $\begin{array}{l}\text { p-value } \\
\text { (overall) }\end{array}$ \\
\hline Beta-blocker & $\begin{array}{l}18 \\
(5.7 \%)\end{array}$ & $\begin{array}{l}8 \\
(4.2 \%)\end{array}$ & $6(8.5 \%)$ & $\begin{array}{l}4 \\
(7.7 \%)\end{array}$ & $\begin{array}{l}0.83 \\
(n / s)\end{array}$ \\
\hline Angiotensin converter inhibitors (ACEi) & $\begin{array}{l}26 \\
(8.3 \%)\end{array}$ & $\begin{array}{l}17 \\
(8.9 \%)\end{array}$ & $4(5.6 \%)$ & $\begin{array}{l}5 \\
(9.6 \%)\end{array}$ & $\begin{array}{l}0.91 \\
(\mathrm{n} / \mathrm{s})\end{array}$ \\
\hline Angiotensin-2 receptor blockers (ARB) & $\begin{array}{l}54 \\
(17.1 \%)\end{array}$ & $\begin{array}{l}30 \\
(15.6 \%)\end{array}$ & $\begin{array}{l}14 \\
(19.7 \%)\end{array}$ & $\begin{array}{l}10 \\
(19.2 \%)\end{array}$ & $\begin{array}{l}0.84 \\
(\mathrm{n} / \mathrm{s})\end{array}$ \\
\hline Loop diuretics & $\begin{array}{l}2 \\
(0.6 \%)\end{array}$ & $\begin{array}{l}1 \\
(0.5 \%)\end{array}$ & 0 & $\begin{array}{l}1 \\
(1.9 \%)\end{array}$ & $\mathrm{n} / \mathrm{a}$ \\
\hline Thiazide diuretics & $\begin{array}{l}26 \\
(8.3 \%)\end{array}$ & $\begin{array}{l}15 \\
(7.8 \%)\end{array}$ & $7(9.9 \%)$ & $\begin{array}{l}4 \\
(7.7 \%)\end{array}$ & $\begin{array}{l}>0.9 \\
(\mathrm{n} / \mathrm{s})\end{array}$ \\
\hline Calcium channel blockers (CCB) & $\begin{array}{l}26 \\
(8.3 \%)\end{array}$ & $\begin{array}{l}13 \\
(6.8 \%)\end{array}$ & $6(8.4 \%)$ & $\begin{array}{l}7 \\
(13.5 \%)\end{array}$ & $\begin{array}{l}0.76 \\
(n / s)\end{array}$ \\
\hline K-sparing diuretics & $\begin{array}{l}3 \\
(0.9 \%)\end{array}$ & $\begin{array}{l}1 \\
(0.5 \%)\end{array}$ & $2(2.8 \%)$ & 0 & $\mathrm{n} / \mathrm{a}$ \\
\hline Statins & $\begin{array}{l}100 \\
(31.7 \%)\end{array}$ & $\begin{array}{l}57 \\
(29.7 \%)\end{array}$ & $\begin{array}{l}18 \\
(25.3 \%)\end{array}$ & $\begin{array}{l}25 \\
(48.1 \%)\end{array}$ & $\begin{array}{l}0.072 \\
(\mathrm{n} / \mathrm{s})\end{array}$ \\
\hline Other lipid lowering agents & $\begin{array}{l}52 \\
(16.5 \%)\end{array}$ & $\begin{array}{l}31 \\
(16.1 \%)\end{array}$ & $\begin{array}{l}12 \\
(16.9 \%)\end{array}$ & $\begin{array}{l}9 \\
(17.3 \%)\end{array}$ & $\mathrm{n} / \mathrm{a}$ \\
\hline Aspirin & $\begin{array}{l}10 \\
(2.8 \%)\end{array}$ & $\begin{array}{l}5 \\
(2.6 \%)\end{array}$ & $2(2.8 \%)$ & $\begin{array}{l}2 \\
(3.8 \%)\end{array}$ & $\mathrm{n} / \mathrm{a}$ \\
\hline Clopidogrel & $\begin{array}{l}3 \\
(0.9 \%)\end{array}$ & $\begin{array}{l}3 \\
(2.6 \%)\end{array}$ & 0 & 0 & $\mathrm{n} / \mathrm{a}$ \\
\hline Xa factor inhibitors & $\begin{array}{l}12 \\
(4.8 \%)\end{array}$ & $\begin{array}{l}8 \\
(4.2 \%)\end{array}$ & $4(5.6 \%)$ & $\begin{array}{l}3 \\
(5.8 \%)\end{array}$ & $\begin{array}{l}>0.9 \\
(\mathrm{n} / \mathrm{s})\end{array}$ \\
\hline Metformin & $\begin{array}{l}63 \\
(20.0 \%)\end{array}$ & $\begin{array}{l}36 \\
(18.7 \%)\end{array}$ & $\begin{array}{l}15 \\
(21.1 \%)\end{array}$ & $\begin{array}{l}12 \\
(23.1 \%)\end{array}$ & $\begin{array}{l}0.88 \\
(n / s)\end{array}$ \\
\hline $\begin{array}{l}\text { Glucagon-like peptide } 1 \text { (GLP1) receptor } \\
\text { analogues (GLP-1Ra) }\end{array}$ & $\begin{array}{l}21 \\
(6.7 \%)\end{array}$ & $\begin{array}{l}12 \\
(6.2 \%)\end{array}$ & $6(8.4 \%)$ & $\begin{array}{l}3 \\
(5.8 \%)\end{array}$ & $\begin{array}{l}0.96 \\
(\mathrm{n} / \mathrm{s})\end{array}$ \\
\hline $\begin{array}{l}\text { Sodium-glucose co-transporter } 2 \text { (SGLT2) } \\
\text { inhibitors (SGLT2i) }\end{array}$ & $\begin{array}{l}24 \\
(7.6 \%)\end{array}$ & $\begin{array}{l}13 \\
(6.8 \%)\end{array}$ & $6(8.4 \%)$ & $\begin{array}{l}5 \\
(9.6 \%)\end{array}$ & $\begin{array}{l}0.94 \\
(\mathrm{n} / \mathrm{s})\end{array}$ \\
\hline $\begin{array}{l}\text { Di-peptyl peptidase } 4 \text { (DPP4) inhibitors } \\
\text { (DPP4i) }\end{array}$ & $\begin{array}{l}15 \\
(4.8 \%)\end{array}$ & $\begin{array}{l}8 \\
(4.2 \%)\end{array}$ & $3(4.2 \%)$ & $\begin{array}{l}4 \\
(7.7 \%)\end{array}$ & $\begin{array}{l}0.95 \\
(\mathrm{n} / \mathrm{s})\end{array}$ \\
\hline Sulfonylureas & $\begin{array}{l}3 \\
(0.9 \%)\end{array}$ & $\begin{array}{l}2 \\
(1.0 \%)\end{array}$ & 0 & $\begin{array}{l}1 \\
(1.9 \%)\end{array}$ & $\mathrm{n} / \mathrm{a}$ \\
\hline
\end{tabular}




\begin{tabular}{|c|c|c|c|c|c|}
\hline Pioglitazone & $\begin{array}{l}5 \\
(1.6 \%)\end{array}$ & $\begin{array}{l}4 \\
(2.1 \%)\end{array}$ & $1(1.4 \%)$ & 0 & $\mathrm{n} / \mathrm{a}$ \\
\hline Insulin & $\begin{array}{l}3 \\
(0.9 \%)\end{array}$ & $\begin{array}{l}2 \\
(1.0 \%)\end{array}$ & $1(1.4 \%)$ & 0 & $\mathrm{n} / \mathrm{a}$ \\
\hline Orlistat & $\begin{array}{l}31 \\
(9.8 \%)\end{array}$ & $\begin{array}{l}21 \\
(10.9 \%)\end{array}$ & $\begin{array}{l}8 \\
(11.3 \%)\end{array}$ & $\begin{array}{l}2 \\
(3.8 \%)\end{array}$ & $\begin{array}{l}0.71 \\
(\mathrm{n} / \mathrm{s})\end{array}$ \\
\hline Levothyroxine & $\begin{array}{l}14 \\
(4.4 \%)\end{array}$ & $\begin{array}{l}10 \\
(5.2 \%)\end{array}$ & $2(2.8 \%)$ & $\begin{array}{l}2 \\
(3.8 \%)\end{array}$ & $\begin{array}{l}0.88 \\
(\mathrm{n} / \mathrm{s})\end{array}$ \\
\hline Testosterone & $\begin{array}{l}23 \\
(7.3 \%)\end{array}$ & $\begin{array}{l}17 \\
(8.8 \%)\end{array}$ & $5(7.0 \%)$ & $\begin{array}{l}1 \\
(1.9 \%)\end{array}$ & $\begin{array}{l}0.74 \\
(\mathrm{n} / \mathrm{s})\end{array}$ \\
\hline $\begin{array}{l}\text { Aromatase inhibitors (anastrozole; letrozole) } \\
\text { or Selective estrogen receptor modulators } \\
\text { (SERMs) }\end{array}$ & $\begin{array}{l}5 \\
(1.6 \%)\end{array}$ & $\begin{array}{l}5 \\
(2.6 \%)\end{array}$ & 0 & 0 & $\mathrm{n} / \mathrm{a}$ \\
\hline Hypnotics & $\begin{array}{l}23 \\
(7.3 \%)\end{array}$ & $\begin{array}{l}12 \\
(6.2 \%)\end{array}$ & $4(5.6 \%)$ & $\begin{array}{l}7 \\
(13.5 \%)\end{array}$ & $\begin{array}{l}0.70 \\
(\mathrm{n} / \mathrm{s})\end{array}$ \\
\hline $\begin{array}{l}\text { Selective serotonin reuptaker inhibitors } \\
\text { (SSRIs) }\end{array}$ & $\begin{array}{l}31 \\
(9.8 \%)\end{array}$ & $\begin{array}{l}15 \\
(7.8 \%)\end{array}$ & $6(8.4 \%)$ & $\begin{array}{l}10 \\
(19.2 \%)\end{array}$ & $\begin{array}{l}0.44 \\
(\mathrm{n} / \mathrm{s})\end{array}$ \\
\hline Other antidepressants and humor stabilizers & $\begin{array}{l}17 \\
(5.4 \%)\end{array}$ & $\begin{array}{l}9 \\
(4.7 \%)\end{array}$ & $6(8.4 \%)$ & $\begin{array}{l}2 \\
(3.8 \%)\end{array}$ & $\begin{array}{l}0.88 \\
(\mathrm{n} / \mathrm{s})\end{array}$ \\
\hline Benzodiazepines & $\begin{array}{l}7 \\
(2.2 \%)\end{array}$ & $\begin{array}{l}4 \\
(2.1 \%)\end{array}$ & $1(1.4 \%)$ & $\begin{array}{l}2 \\
(3.8 \%)\end{array}$ & $\begin{array}{l}>0.9 \\
(\mathrm{n} / \mathrm{s})\end{array}$ \\
\hline Atypical antipsychotics & $\begin{array}{l}2 \\
(0.6 \%)\end{array}$ & $\begin{array}{l}2 \\
(1.0 \%)\end{array}$ & 0 & 0 & $\mathrm{n} / \mathrm{a}$ \\
\hline Central nervous system (CNS) stimulants & $\begin{array}{l}38 \\
(12.1 \%)\end{array}$ & $\begin{array}{l}23 \\
(12.0 \%)\end{array}$ & $7(9.9 \%)$ & $\begin{array}{l}8 \\
(15.4 \%)\end{array}$ & $(n / s)$ \\
\hline Alpha-1 adrenergic blockers & $\begin{array}{l}10 \\
\text { (3.2\%) }\end{array}$ & $\begin{array}{l}5 \\
(2.6 \%)\end{array}$ & $2(2.8 \%)$ & $\begin{array}{l}3 \\
(5.8 \%)\end{array}$ & $\begin{array}{l}>0.9 \\
(\mathrm{n} / \mathrm{s})\end{array}$ \\
\hline Phosphodiesterase 5 inhibitors & $\begin{array}{l}33 \\
(10.5 \%)\end{array}$ & $\begin{array}{l}17 \\
(8.9 \%)\end{array}$ & $\begin{array}{l}8 \\
(11.3 \%)\end{array}$ & $\begin{array}{l}8 \\
(15.4 \%)\end{array}$ & $\begin{array}{l}0.76 \\
\text { (n/s) }\end{array}$ \\
\hline Omega 3 & $\begin{array}{l}13 \\
(4.1 \%)\end{array}$ & $\begin{array}{l}8 \\
(4.2 \%)\end{array}$ & $2(2.8 \%)$ & $\begin{array}{l}3 \\
(5.8 \%)\end{array}$ & $\begin{array}{l}>0.9 \\
(\mathrm{n} / \mathrm{s})\end{array}$ \\
\hline Vitamin D & $\begin{array}{l}42 \\
(13.3 \%)\end{array}$ & $\begin{array}{l}23 \\
(12.0 \%)\end{array}$ & $\begin{array}{l}10 \\
(14.1 \%)\end{array}$ & $\begin{array}{l}9 \\
(17.3 \%)\end{array}$ & $\begin{array}{l}>0.9 \\
(\mathrm{n} / \mathrm{s})\end{array}$ \\
\hline Zinc & $\begin{array}{l}12 \\
(3.8 \%)\end{array}$ & $\begin{array}{l}7 \\
(3.6 \%)\end{array}$ & $4(5.6 \%)$ & $\begin{array}{l}1 \\
(1.9 \%)\end{array}$ & $\begin{array}{l}>0.8 \\
(\mathrm{n} / \mathrm{s})\end{array}$ \\
\hline Biotin & $\begin{array}{l}9 \\
(2.9 \%)\end{array}$ & $\begin{array}{l}1 \\
(0.5 \%)\end{array}$ & $3(4.2 \%)$ & $\begin{array}{l}5 \\
(9.6 \%)\end{array}$ & $\begin{array}{l}0.58 \\
(\mathrm{n} / \mathrm{s})\end{array}$ \\
\hline Vitamin C & $\begin{array}{l}27 \\
(8.6 \%)\end{array}$ & $\begin{array}{l}18 \\
(9.4 \%)\end{array}$ & $7(9.9 \%)$ & $\begin{array}{l}2 \\
(3.8 \%)\end{array}$ & $\begin{array}{l}>0.9 \\
(\mathrm{n} / \mathrm{s})\end{array}$ \\
\hline
\end{tabular}


$\mathrm{AGA}=$ androgenetic alopecia; $5 \mathrm{ARi}=$ Androgen receptor inhibitors; $\mathrm{n} / \mathrm{s}=$ non-significant; $\mathrm{n} / \mathrm{a}=$ nonapplicable

${ }^{*} p<0.0001$ vs AGA no-ARi males

${ }^{\#} \mathrm{p}<0.0001$ vs AGA-ARi males

$\&_{p}<0.05$ vs no-5ARi users (non-AGA and AGA no-5ARi)

Table 5. Vaccines and lifestyle.

\begin{tabular}{|c|c|c|c|c|c|}
\hline Vaccine and lifestyle & $\begin{array}{l}\text { Overall } \\
\text { males } \\
(n=305)\end{array}$ & $\begin{array}{l}\text { Non-AGA } \\
\text { males } \\
(n=192)\end{array}$ & $\begin{array}{l}\text { AGA no-5ARi } \\
\text { males } \\
(n=71)\end{array}$ & $\begin{array}{l}\text { AGA- } \\
5 A R i \\
(n=52)\end{array}$ & $\begin{array}{l}\text { p-value } \\
\text { (overall) }\end{array}$ \\
\hline Vaccine - BCG & $\begin{array}{l}305 \\
(100 \%)\end{array}$ & $192(100 \%)$ & $71(100 \%)$ & $\begin{array}{l}52 \\
(100 \%)\end{array}$ & $\begin{array}{l}1.0 \\
(n / s)\end{array}$ \\
\hline Vaccine - Influenza (2020) & $\begin{array}{l}44 \\
(14.0 \%)\end{array}$ & $26(13.5 \%)$ & $11(15.5 \%)$ & $\begin{array}{l}7 \\
(13.5 \%)\end{array}$ & $\begin{array}{l}>0.9 \\
(\mathrm{n} / \mathrm{s})\end{array}$ \\
\hline $\begin{array}{l}\text { Vaccine - Pneumococcal } \\
\text { (since 2017) }\end{array}$ & $\begin{array}{l}34 \\
(10.8 \%)\end{array}$ & $18(9.4 \%)$ & $10(14.1 \%)$ & 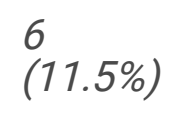 & $\begin{array}{l}0.84 \\
(n / s)\end{array}$ \\
\hline Current smoking & $10(3.2 \%)$ & $6(3.1 \%)$ & $2(2.8 \%)$ & $2(3.8 \%)$ & $\begin{array}{l}>0.9 \\
(\mathrm{n} / \mathrm{s})\end{array}$ \\
\hline Regular physical activity & $\begin{array}{l}98 \\
(31.1 \%)\end{array}$ & $56(29.2 \%)$ & $20(28.2 \%)$ & $\begin{array}{l}22 \\
(42.3 \%)\end{array}$ & $\begin{array}{l}0.31 \\
(n / s)\end{array}$ \\
\hline
\end{tabular}

AGA = androgenetic alopecia; $5 \mathrm{ARi}=$ Androgen receptor inhibitors; $n / s=$ non-significant; $n / a=$ nonapplicable

\section{COVID-19 characterization and proposed treatment}

Tables 6 to 9 detail the characterization of COVID-19 and proposed treatments. Table 6 describes the rates of each cluster of clinical manifestations. Table 7 depicts the mean presence, duration, and time-toappearance of each symptom. Table 8 describes the major drugs used as proposed anti-COVID treatments and Table 9 describes additional drugs and supplements prescribed at an individual basis.

In regards with COVID-19 types of clinical presentation (Table 6), anosmia-ageusia dominance was more prevalent in non-AGA than AGA no-5ARi males. Dengue-like manifestations were more present in AGA no5ARi than 5ARi males, and more present in 5ARi users than non-users. URTI-like symptoms were more present in non-AGA and AGA no-5ARi users than ARI-5ARi males. Mixed and unspecific presentations 
were similarly present in all groups. The majority of 5ARi users were either asymptomatic or presented anosmia and/or ageusia as the most remarkable or only clinical symptom.

Clinical manifestations presented wide differences in terms of percentage of presence, time to appearance and duration for multiple symptoms (Table 7). In terms of percentage of male patients with COVID-19 presenting each symptom, those present in more than $50 \%$ include anosmia $(68.9 \%)$ and ageusia (61.2\%). Those presented in between one quarter and half of COVID-19 males include headache (37.5\%), hyporexia (37.5\%), fatigue (35.2\%), dry cough (35.2\%), fever or "feverish" (33.9\%), thoracic pain (32.4\%), conjunctival hyperemia (29.5\%), weakness (29.5\%), nasal congestion or rhinorrhea $(28.6 \%$ and myalgia ( $26.3 \%$. Symptoms present in $10 \%$ to $25 \%$ of patients include "sore throat" (23.5\%, "feverish" alone (22.5\%), diarrhea (20.9\%), dizziness (17.5\%), upper back pain (14.0\%) and fever alone $(11.4 \%)$. Symptoms present in less than $10 \%$ but in a non-negligeble number of patients include arthralgia (9.2\%), shortness of breath (6.3\%), "sinusitis" (6.3\%), nauseas (5.7\%). lower back pain (4.1\%), abdominal pain (4.1\%) and pre-orbital pain (3.5\%).

Symptoms that appeared earlier in COVID-19 (< 1.6 day from the first symptom; in days) include nasal congestion or rhinorrhea $-1.0 \pm 0.1$, nauseas $-1.3 \pm 0.7$, pre-orbital pain $-1.3 \pm 0.6$, "feverish" $-1.4 \pm$ 0.8 , fever $-1.4 \pm 0.7$, arthralgia $-1.4 \pm 0.6$, abdominal pain $-1.4 \pm 0.7$, dizziness $-1.5 \pm 0.9$, weakness $-1.5 \pm 0.9$, conjunctival hyperemia $-1.6 \pm 0.9$, headache $-1.6 \pm 0.9$, "sinusitis" $-1.6 \pm 0.8$, fatigue -1.6 \pm 0.9 and myalgia $-1.6 \pm 0.9$. Later symptoms (> 3.5 days since first symptom) include anosmia $-3.8 \pm$ 1.4 , ageusia $-3.9 \pm 1.4$ and shortness of breath $-4.4 \pm 1.1$ (Table 7 ).

At least in patients treated for COVID-19 (Table 7), symptoms with lower duration (< 3.1 days) include dizziness $-1.7 \pm 1.0$, shortness of breath $-2.3 \pm 0.9$, arthralgia $-2.5 \pm 1.2$, abdominal pain $-2.6 \pm 1.3$, nauseas $-2.8 \pm 1.2$, "feverish" $-2.9 \pm 1.3$, fever $-3.1 \pm 1.4$ and weakness $-3.1 \pm 2.2$. Symptoms with intermediate duration ( 3.1 to 5 days) include diarrhea $-3.2 \pm 1.9$, myalgia $-3.6 \pm 1.8$, pre-orbital pain $3.6 \pm 1.6$, lower back pain $-3.9 \pm 1.5$, nasal congestion or rhinorrhea $-4.0 \pm 2.0$, hyporexia $-4.3 \pm 2.0$, thoracic pain $-4.3 \pm 2.2$, upper back pain $-4.3 \pm 1.8$ and conjunctival hyperemia $-4.9 \pm 2.1$. Symptoms that persist for longer periods of time (> 5 days) include dry cough $-5.3 \pm 2.4$, headache $-5.5 \pm 2.4$, "sore throat" $-5.5 \pm 1.9$, "sinusitis" - $7.2 \pm 2.6$, ageusia $-7.4 \pm 5.1$, anosmia $-7.9 \pm 5.5$ and fatigue -8.4 \pm 4.6 .

Besides being highly heterogeneous, rate, duration and time-to-appearance of symptoms varied particularly according to the presence of male AGA and use of dutasteride.

Among 5ARi users, fever, shortness of breath, "sinusitis", dizziness, myalgia, arthralgia, thoracic pain, lower back pain were absent, while only one patient presented "feverish", nasal congestion or rhinorrhea, "sore throat", fatigue, weakness, upper back pain, nauseas, vomiting, abdominal pain and pre-orbital pain, and two patients that presented headache, dry cough, diarrhea and conjunctival hyperemia (Table 7). The only symptoms that were not negligeable among 5ARi users were anosmia and ageusia. In common, all symptoms were significantly less present in AGA-ARi males compared to non-AGA, AGA no-ARi, and overall ARi males, except for nauseas, vomiting, abdominal pain, and pre-orbital pain, since these were 
also present in very few patients in all groups. Because of the lack of symptomatology, except for anosmia and ageusia, time of appearance and duration were only feasible to be compared between nonAGA and AGA no-5ARi males (Table 7).

Fever was present in similar patterns between non-AGA and AGA no-5ARi males. Although presence of feeling of fever with no fever confirmed ("feverish") was also similar between these two groups, in nonAGA males duration was slightly but significantly lower than AGA no-5ARi. Nasal congestion and rhinorrhea had the same patterns and difference in terms of duration compared to "feverish". Non-AGA had less and lower duration of headache than AGA no-5ARi males, while time to appearance was similar between them. Shortness of breath had similar presence rate, time to appearance and duration between non-AGA and AGA no-5ARi males.

Anosmia was present in a similar percentage of non-AGA and AGA no-5ARi males, and more present in these two groups than in 5ARi users. Time to appearance and duration of anosmia was significantly higher in AGA no-5ARi than non-AGA and AGA-5ARi, and significantly higher in non-AGA than AGA-5ARi males. Ageusia had identical features than anosmia in terms of presence, time to appearance and duration, when compared to anosmia, and when compared between groups.

Dry cough was more present and had longer duration in AGA no-5ARi than non-AGA males, while time to appearance was similar between them. Self-reported perception of "sinusitis" and "sore throat" was equally present in non-AGA and AGA no-5ARi males, developed with similar intervals between them, whereas duration of both was longer in the AGA no-5ARi group.

Dizziness was present at similar extent and duration between non-AGA and AGA no-5ARi males. Conversely, fatigue was more present and persisted for longer periods in AGA no-5ARi compared to nonAGA, while weakness was equally present but had longer duration in AGA no-5ARi males. Myalgia affected more severely AGA no-5ARi than non-AGA males, in terms of presence and duration. Arthralgia was more present in AGA no-5ARi males, but had similar time-to-appearance and duration than non-AGA.

Thoracic, upper back, and lower back pain affected a higher percentage of AGA no-5ARi than non-AGA males, and had similar time-to-appearance and duration between them, except for a slightly higher time until appearance in non-AGA males.

Diarrhea, nauseas, vomiting, and abdominal pain were present in similar percentage and had similar duration between non-AGA and AGA no-ARi males. Conjunctival hyperemia was more commonly present and lasted for longer periods in AGA no-5ARi, compared to non-AGA males, while pre-orbital pain was similarly present between these groups.

In regards with proposed therapeutical options for COVID-19, all patients received azithromycin. Nitazoxanide was associated in $72.4 \%$, at higher percentage of males from the non-AGA and AGA no-5ARi groups, compared to 5ARi users. Hydroxychloroquine and ivermectin were prescribed for $21.6 \%$ and $14.6 \%$ of patients, respectively, equally between groups. Among non-chronic 5ARi users, dutasteride was 
significantly more used in AGA than non-AGA males, while spironolactone was used in similar proportions (Table 8).

There were no significant differences between the percentage of patients treated with any additional drugs of supplements for COVID-19. Warfarin, acetylsalicylic acid (ASA), bromhexine, N-acetyl-cysteine and colchicine have not been added as therapies for COVID-19 to any, or until to two patients.

Table 6. Clinical clustering 


\begin{tabular}{|c|c|c|c|c|c|}
\hline Clinical clustering & $\begin{array}{l}\text { Overall } \\
\text { males } \\
(n= \\
305)\end{array}$ & $\begin{array}{l}\text { Non-AGA males } \\
(n=192)\end{array}$ & $\begin{array}{l}\text { AGA no-5ARi } \\
\text { males } \\
(n=71)\end{array}$ & $\begin{array}{l}\text { AGA-5ARi } \\
(n=52)\end{array}$ & $\begin{array}{l}\text { p-value } \\
\text { (overall) }\end{array}$ \\
\hline $\begin{array}{l}\text { Anosmia-Ageusia } \\
\text { dominance } \\
\text { (p-value) }\end{array}$ & $\begin{array}{l}69 \\
(21.9 \%)\end{array}$ & $\begin{array}{l}56(29.2 \%) \\
(p=0.01 \text { vs AGA no- } \\
A R i) \\
(n / s(0.082) \text { vs } \\
A G A-5 A R i)\end{array}$ & $\begin{array}{l}6(8.5 \%) \\
(n / s \text { vs } A G A- \\
5 A R i)\end{array}$ & $\begin{array}{l}7(13.5 \%) \\
(n / s \text { vs no- } \\
5 A R i)\end{array}$ & $\begin{array}{l}< \\
0.0001\end{array}$ \\
\hline $\begin{array}{l}\text { Dengue-like } \\
\text { (p-value) }\end{array}$ & $\begin{array}{l}56 \\
(17.8 \%)\end{array}$ & $\begin{array}{l}36(18.7 \%) \\
(n / s \text { vs } A G A \text { no- } \\
\text { ARi) } \\
(n / s(p=0.063) v s \\
A G A-5 A R i)\end{array}$ & $\begin{array}{l}19(26.8 \%) \\
(p=0.019 \mathrm{vs} \\
A G A-5 A R i)\end{array}$ & $\begin{array}{l}1(1.9 \%) \\
(p=0.035 \text { vs } \\
\text { no-5ARi) }\end{array}$ & 0.003 \\
\hline $\begin{array}{l}\text { URTI-like } \\
\text { (p-value) }\end{array}$ & $\begin{array}{l}102 \\
(32.4 \%)\end{array}$ & $\begin{array}{l}66(34.4 \%) \\
(n / s(p=0.063) v s \\
A G A \text { no-ARi) } \\
(p=0.0003 \text { vs } A G A- \\
5 A R i)\end{array}$ & $\begin{array}{l}35(49.3 \%) \\
(p<0.0001 \text { vs } \\
A G A-5 A R i)\end{array}$ & $\begin{array}{l}1(1.9 \%) \\
(p<0.0001 \text { vs } \\
\text { no-5ARi) }\end{array}$ & $\begin{array}{l}< \\
0.0001\end{array}$ \\
\hline $\begin{array}{l}\text { GI infection-like } \\
\text { (p-value) }\end{array}$ & $\begin{array}{l}24 \\
(7.6 \%)\end{array}$ & $\begin{array}{l}14(7.3 \%) \\
\text { (n/s vs AGA no- } \\
\text { ARi) } \\
(n / s \text { vs AGA-5ARi) }\end{array}$ & $\begin{array}{l}9(12.7 \%) \\
(n / s \text { vs } A G A- \\
5 A R i)\end{array}$ & $\begin{array}{l}1(1.9 \%) \\
(n / s \text { vs no- } \\
5 A R i)\end{array}$ & $\begin{array}{l}0.082 \\
(n / s)\end{array}$ \\
\hline $\begin{array}{l}\text { Mixed } \\
\text { (p-value) }\end{array}$ & $\begin{array}{l}22 \\
(7.6 \%)\end{array}$ & $\begin{array}{l}13 \text { (6.8\%) } \\
\text { (n/s vs AGA no- } \\
\text { ARi) } \\
(n / s \text { vs AGA-5ARi) }\end{array}$ & $\begin{array}{l}9(15.5 \%) \\
(n / s \text { vs } A G A- \\
5 A R i)\end{array}$ & $\begin{array}{l}0 \\
(n / s v s \text { vo- } \\
5 A R i)\end{array}$ & $\begin{array}{l}0.32 \\
(\mathrm{n} / \mathrm{s})\end{array}$ \\
\hline $\begin{array}{l}\text { Unspecific } \\
\text { (p-value) }\end{array}$ & $\begin{array}{l}35 \\
(11.1 \%)\end{array}$ & $\begin{array}{l}23(12.0 \%) \\
(n / s \text { vs AGA no- } \\
\text { ARi) } \\
(n / s \text { vs AGA-5ARi) }\end{array}$ & $\begin{array}{l}12(16.9 \%) \\
(n / s \text { vs } A G A- \\
5 A R i)\end{array}$ & $\begin{array}{l}0 \\
(n / s \text { vs no- } \\
5 A R i)\end{array}$ & 0.011 \\
\hline $\begin{array}{l}\text { Asymptomatic } \\
\text { (p-value) }\end{array}$ & $\begin{array}{l}51 \\
(16.2 \%)\end{array}$ & $\begin{array}{l}9(4.7 \%) \\
(n / s \text { vs } A G A \text { no- } \\
\text { ARi) } \\
(p<0.0001 \text { vs } A G A- \\
5 A R i)\end{array}$ & $\begin{array}{l}0 \\
(p<0.0001 \text { vs } \\
A G A-5 A R i)\end{array}$ & $\begin{array}{l}42(80.8 \%) \\
(p<0.0001 \text { vs } \\
\text { no-5ARi) }\end{array}$ & $\begin{array}{l}< \\
0.0001\end{array}$ \\
\hline
\end{tabular}


URTI = Upper respiratory tract infection; GI = Gastrointestinal; AGA = androgenetic alopecia; 5ARi = Androgen receptor inhibitors; $\mathrm{n} / \mathrm{s}=$ non-significant; $\mathrm{n} / \mathrm{a}=$ non-applicable

Table 7. Clinical manifestations in COVID-19: presence (\%), time-to-appearance and duration. 


\begin{tabular}{|c|c|c|c|c|c|}
\hline $\begin{array}{l}\text { Clinical } \\
\text { manifestations }\end{array}$ & $\begin{array}{l}\text { Overall } \\
\text { males } \\
(n= \\
305)\end{array}$ & $\begin{array}{l}\text { Non-AGA males } \\
(n=192)\end{array}$ & $\begin{array}{l}\text { AGA no-5ARi } \\
\text { males } \\
(n=71)\end{array}$ & $\begin{array}{l}\text { AGA-5ARi } \\
(n=52)\end{array}$ & $\begin{array}{l}\text { p-value } \\
\text { (overall) }\end{array}$ \\
\hline
\end{tabular}

\section{Fever}

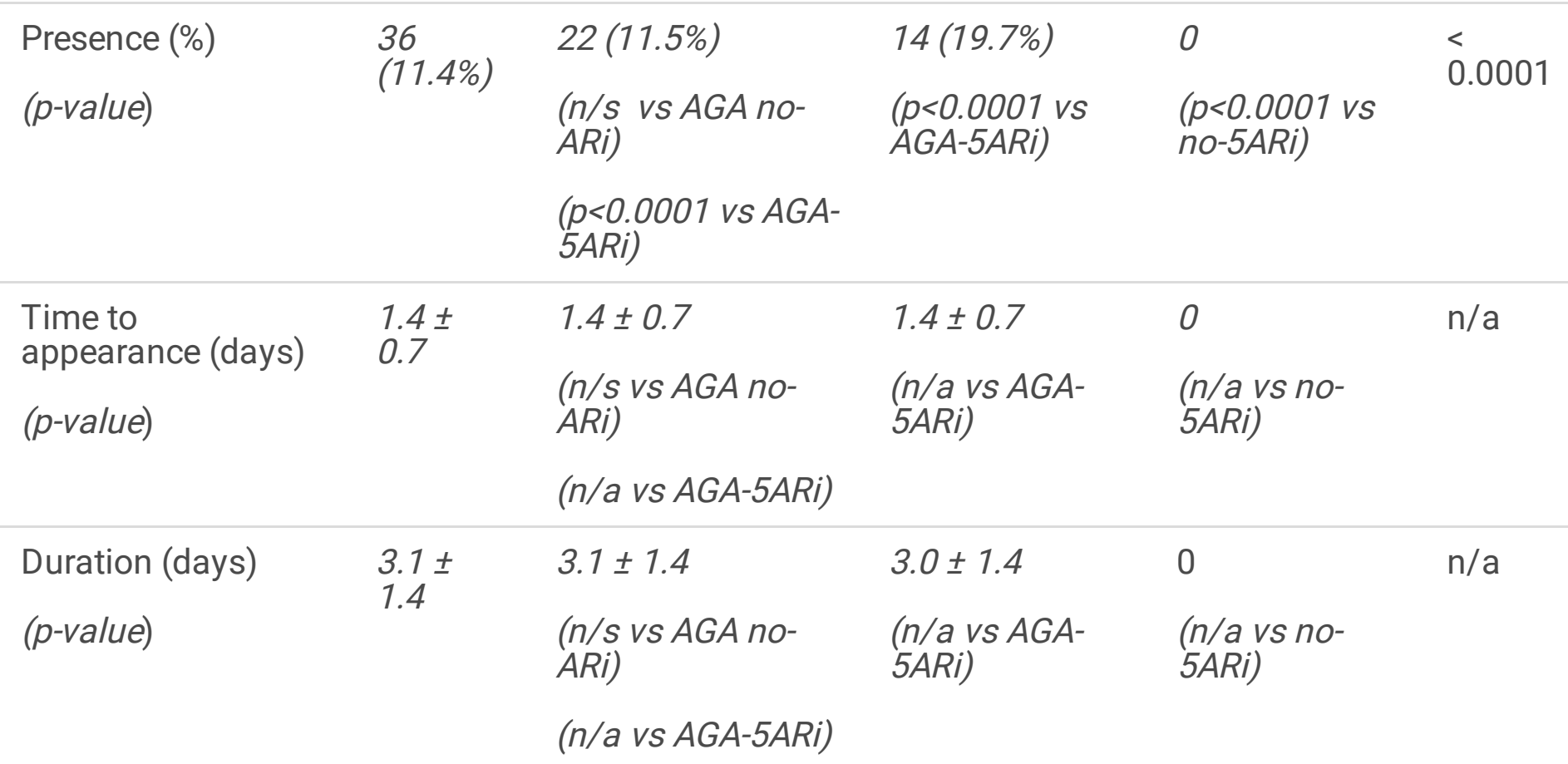

"Feverish"

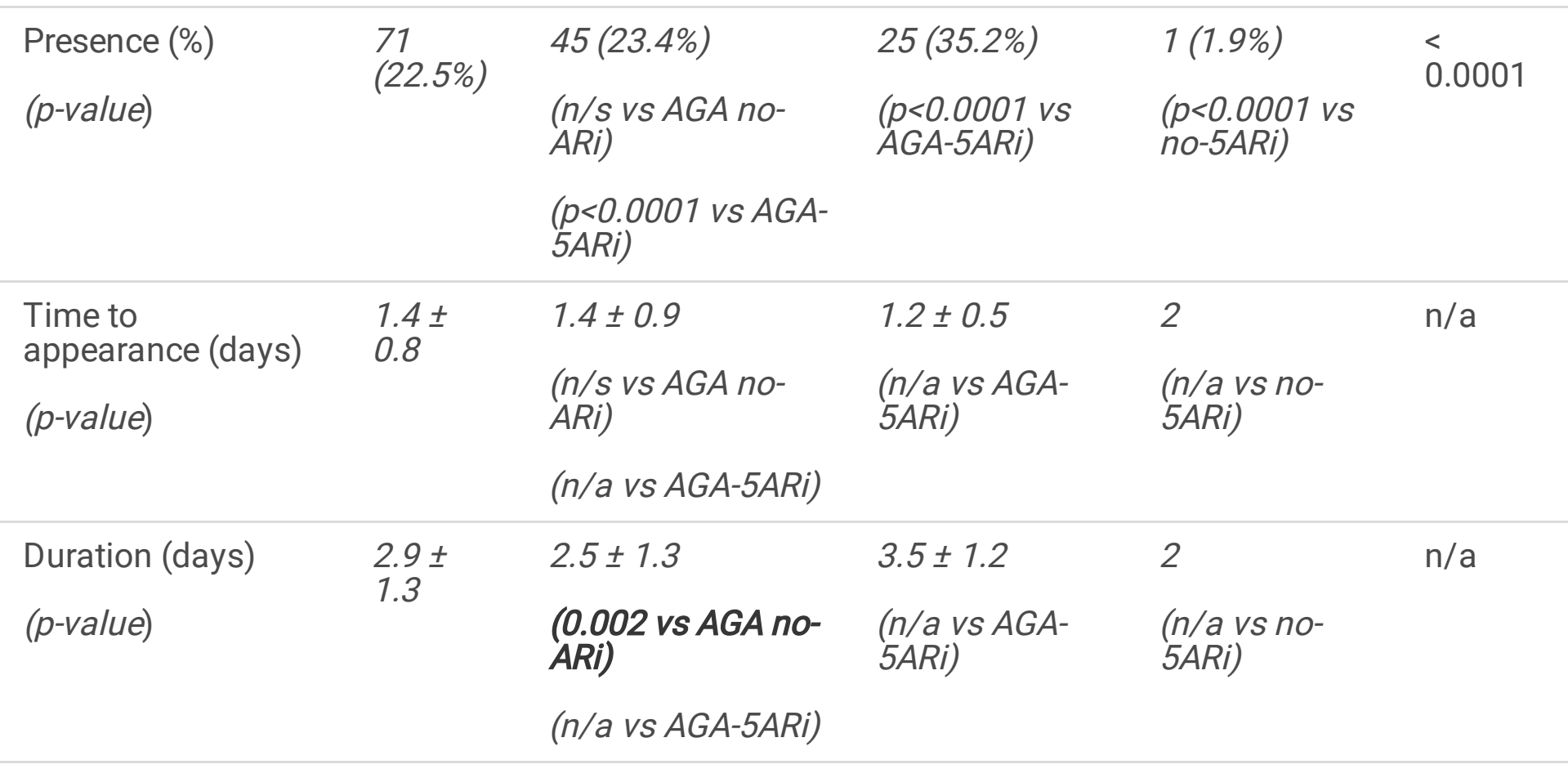


or rhinorrhea

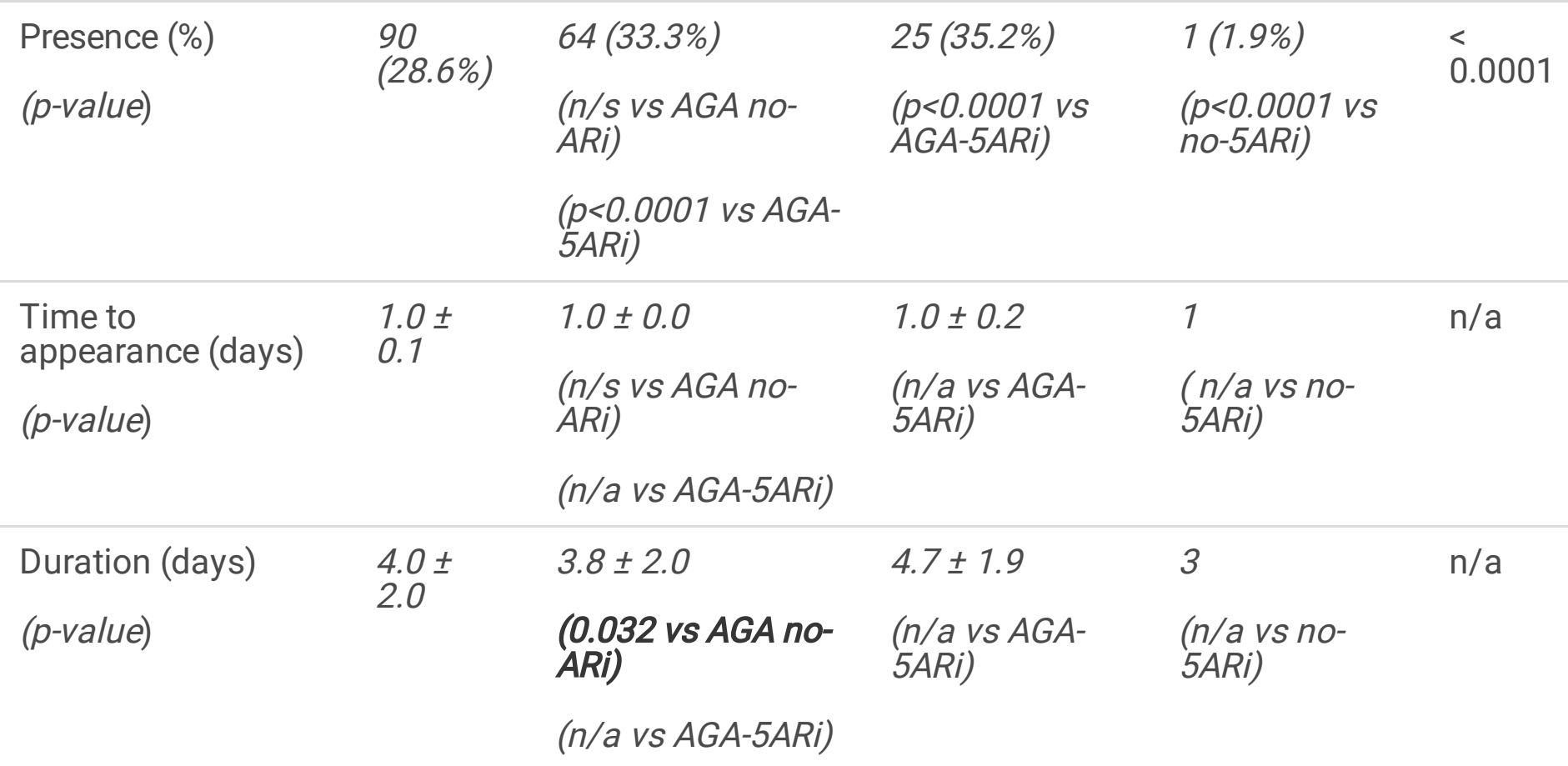

\section{Headache}

$\begin{array}{lll}\text { Presence (\%) } & 118 & 70(36.5 \%) \\ \text { (p-value) } & (37.5 \%) & (p=0.0004 \text { vs } A G A \\ & & \text { no-ARi) } \\ & & (p<0.0001 \text { vs } A G A- \\ & & 5 A R i)\end{array}$

Time to

appearance (days)

(p-value)

$1.6 \pm \quad 1.6 \pm 0.9$

0.9

(n/s vs AGA no-

ARi)

(n/a vs AGA-5ARi)

Duration (days)

(p-value)
$5.5 \pm$

2.4
$5.0 \pm 2.1$

( $p<0.0001$ vs $A G A$ no-ARi)

(n/a vs AGA-5ARi)
46 (64.8\%)

(p<0.0001 vs

AGA-5ARi)
$2(3.8 \%)$

( $p<0.0001$ vs

no-5ARi)
$<$

0.0001
$1.6 \pm 1.0$

(n/a vs AGA-

5ARi)

$6.2 \pm 2.8$

(n/a vs AGA-

5ARi)
$1.5 \pm 0.5(1 ; 2) \quad n / a$

(n/a vs no-

5ARi)
$3.5 \pm 0.5(3 ; 4) \quad n / a$

(n/a vs no-

5ARi)

\section{Shortness of breath}

Presence (\%)

(p-value)
20

(6.3\%)

$10(5.2 \%)$

(n/s vs AGA no-

ARi)
$10(14.1 \%)$

$(p<0.0001$ vs AGA-5ARi)
0

$(p<0.0001$ vs no-5ARi) 
( $p<0.0001$ vs AGA-

5ARi)

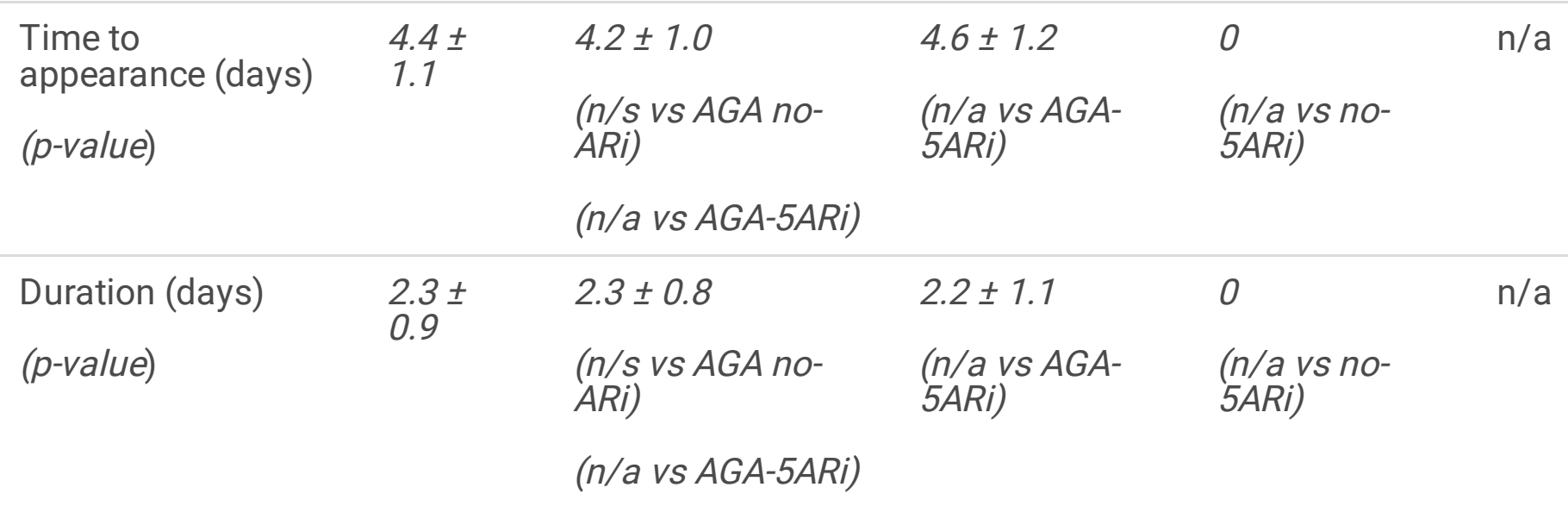

\section{Anosmia}

$\begin{array}{lll}\text { Presence (\%) } & 217 & 147(76.6 \%) \\ \text { (p-value) } & \text { (68.9\%) } & (n / s \text { vs AGA no- } \\ & & \text { ARi) } \\ & & (p<0.0001 \text { vs } A G A- \\ & & 5 \text { ARi) }\end{array}$

Time to

appearance (days) $\quad 1.4$

(p-value)

$3.8 \pm$
1.4

$3.5 \pm 1.3$

( $p<0.0001$ vs AGA no-ARi)

( $p=0.009$ vs $A G A-$ 5ARi)

$\begin{array}{lll}\text { Duration (days) } & 7.9 \pm & 7.2 \pm 4.9 \\ \text { (p-value) } & 5.5^{ \pm} & (p=0.0005 \text { vs } A G A \\ & & \text { no-ARi) } \\ & & (p=0.012 \text { vs } A G A- \\ & & 5 A R i)\end{array}$

$63(88.7 \%)$

( $p<0.0001$ vs AGA-5ARi)
$7(13.5 \%)$

$(p<0.0001$ vs no-5ARi)
$<$

0.0001

$4.7 \pm 1.0$

$(p=0.009$ vs

AGA-5ARi)

$2 \pm 1.1$

$<$

$(p=0.0002$ vs

0.0001 no-5ARi)

\section{Ageusia}

Presence (\%)

( $p$-value)
204

(61.2\%)
134 (69.8\%)

( $n / s$ vs AGA no-

ARi)

( $p<0.0001$ vs $A G A-$

5ARi)

\section{Time to}

appearance (days)

(p-value)
$3.9 \pm$

1.4

$3.6 \pm 1.4$

( $p<0.0001$ AGA no-ARi)
$10.1 \pm 6.4$

$(p=0.001$ vs

AGA-5ARi)
$3.7 \pm 1.2$

$(p=0.005 v s$ no-5ARi)
$<$

0.0001 


\begin{tabular}{|c|c|c|c|c|}
\hline $\begin{array}{l}\text { Duration (days) } \\
\text { (p-value) }\end{array}$ & $\begin{array}{l}7.4 \pm \\
5.1\end{array}$ & $\begin{array}{l}6.7 \pm 4.6 \\
(p=0.0004 \text { vs } A G A \\
\text { no-ARi) }\end{array}$ & $\begin{array}{l}9.5 \pm 5.7 \\
(p=0.003 \text { vs } \\
\text { AGA-5ARi) }\end{array}$ & $\begin{array}{l}3.0 \pm 0.6 \\
(p=0.0055 \mathrm{vs} \\
\text { no-5ARi) }\end{array}$ \\
\hline & & $\begin{array}{l}(p=0.008 \text { vs } A G A- \\
5 A R i)\end{array}$ & & \\
\hline
\end{tabular}

\section{Dry cough}

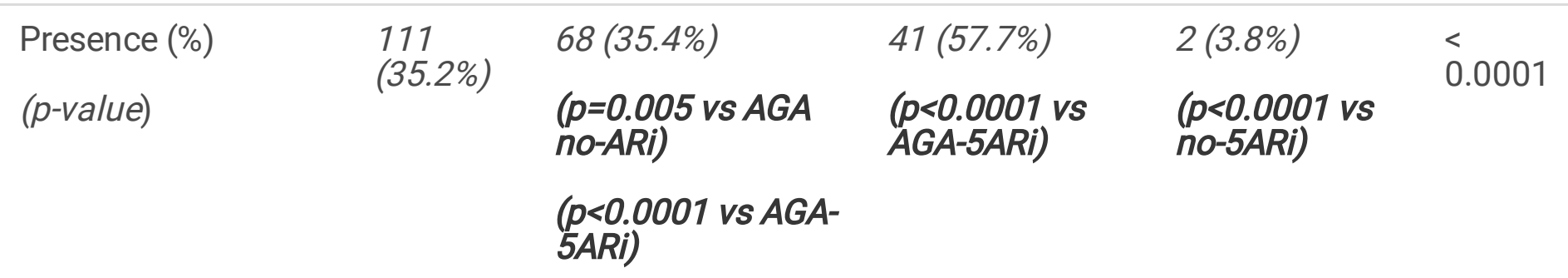

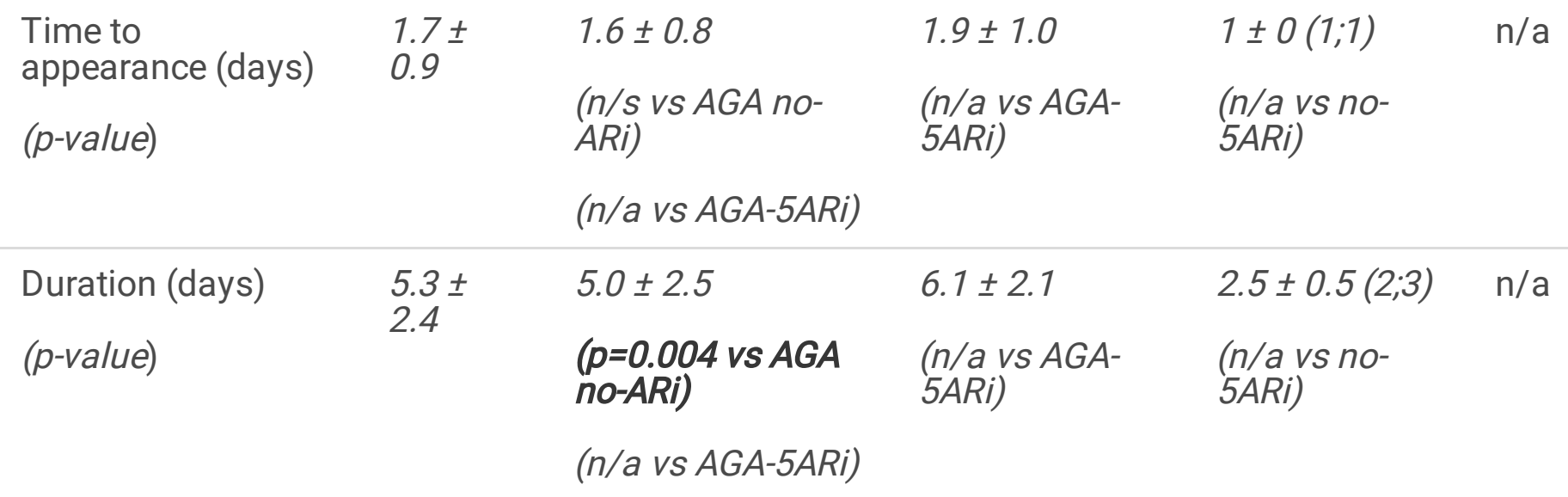

\section{"Sinusitis"}

\begin{tabular}{|c|c|c|}
\hline $\begin{array}{l}\text { Presence (\%) } \\
(p \text {-value) }\end{array}$ & $\begin{array}{l}20 \\
(6.3 \%)\end{array}$ & $\begin{array}{l}12(6.2 \%) \\
(n / s \text { vs } A G A \text { no- } \\
\text { ARi) }\end{array}$ \\
\hline & & $\begin{array}{l}(p<0.0001 \text { vs } A G A- \\
5 A R i)\end{array}$ \\
\hline
\end{tabular}

\begin{tabular}{|c|c|c|c|c|}
\hline $\begin{array}{l}\text { Time to } \\
\text { appearance (days) } \\
\text { (p-value) }\end{array}$ & $\begin{array}{l}1.6 \pm \\
0.8\end{array}$ & $\begin{array}{l}1.5 \pm 0.7 \\
(\mathrm{n} / \mathrm{s} \text { vs } A G A \text { no- } \\
\text { ARi })\end{array}$ & $\begin{array}{l}1.7 \pm 1.0 \\
(n / a \text { vs } A G A- \\
5 A R i)\end{array}$ & $\begin{array}{l}0 \\
(n / a \text { vs no- } \\
5 A R i)\end{array}$ \\
\hline
\end{tabular}

(n/a vs AGA-5ARi)

$\begin{array}{lllll}\text { Duration (days) } & 7.2 \pm & 5.8 \pm 2.3 & 9.0 \pm 1.9 & 0 \\ \text { (p-value) } & 2.6 & \begin{array}{l}(p=0.0004 \text { vs } A G A \\ \text { no-ARi) }\end{array} & \begin{array}{l}\text { (n/a vs } A G A- \\ \text { 5ARi) }\end{array} & \begin{array}{l}\text { (n/a vs no- } \\ \text { 5ARi) }\end{array}\end{array}$

$8(11.3 \%)$

$(p<0.0001$ vs $\quad(p<0.0001$ vs

AGA-5ARi)

0.0001 
"Sore throat"

\begin{tabular}{|c|c|c|c|c|c|}
\hline $\begin{array}{l}\text { Presence (\%) } \\
\text { (p-value) }\end{array}$ & $\begin{array}{l}74 \\
(23.5 \%)\end{array}$ & $\begin{array}{l}50(26.0 \%) \\
(\mathrm{n} / \mathrm{s} \text { vs } A G A \text { no- } \\
\text { ARi) }\end{array}$ & $\begin{array}{l}23(32.4 \%) \\
(p<0.0001 \text { vs } \\
A G A-5 A R i)\end{array}$ & $\begin{array}{l}1(1.9 \%) \\
(p<0.0001 \text { vs } \\
\text { no-5ARi) }\end{array}$ & $\begin{array}{l}< \\
0.0001\end{array}$ \\
\hline & & $\begin{array}{l}(p<0.0001 \text { vs } A G A- \\
5 A R i)\end{array}$ & & & \\
\hline $\begin{array}{l}\text { Time to } \\
\text { appearance (days) } \\
\text { (p-value) }\end{array}$ & $\begin{array}{l}2.0 \pm \\
1.0\end{array}$ & $\begin{array}{l}1.9 \pm 1.0 \\
(\mathrm{n} / \mathrm{s} \text { vs } A G A \text { no- } \\
\text { ARi) } \\
\text { (n/a vs AGA-5ARi) }\end{array}$ & $\begin{array}{l}2.2 \pm 1.2 \\
(n / a \text { vs } A G A- \\
5 A R i)\end{array}$ & $\begin{array}{l}2 \\
(n / a \text { vs no- } \\
5 A R i)\end{array}$ & $\mathrm{n} / \mathrm{a}$ \\
\hline $\begin{array}{l}\text { Duration (days) } \\
\text { (p-value) }\end{array}$ & $\begin{array}{l}5.5 \pm \\
1.9\end{array}$ & $\begin{array}{l}5.2 \pm 1.8 \\
(p=0.029 \text { vs } A G A \\
\text { no-ARi) } \\
\text { (n/a vs } A G A-5 A R i)\end{array}$ & $\begin{array}{l}6.3 \pm 1.9 \\
(n / a \text { vs } A G A- \\
5 A R i)\end{array}$ & $\begin{array}{l}4 \\
(n / a \text { vs no- } \\
5 A R i)\end{array}$ & $\mathrm{n} / \mathrm{a}$ \\
\hline
\end{tabular}

\section{Dizziness}

$\begin{array}{llllll}\text { Presence }(\%) & 55 & 24(12.5 \%) & 31(43.7 \%) & 0 & < \\ \text { (p-value }) & (17.5 \%) & (n / s \text { vs } A G A \text { no- } & \begin{array}{l}\text { (p<0.0001 vs } \\ \text { AGA-5ARi) }\end{array} & \begin{array}{l}\text { (p<0.0001 vs } \\ \text { no-5ARi) }\end{array} & 0.0001 \\ & & & & \\ & & & & \\ & & (p=0.0001 \text { vs } & & & \end{array}$

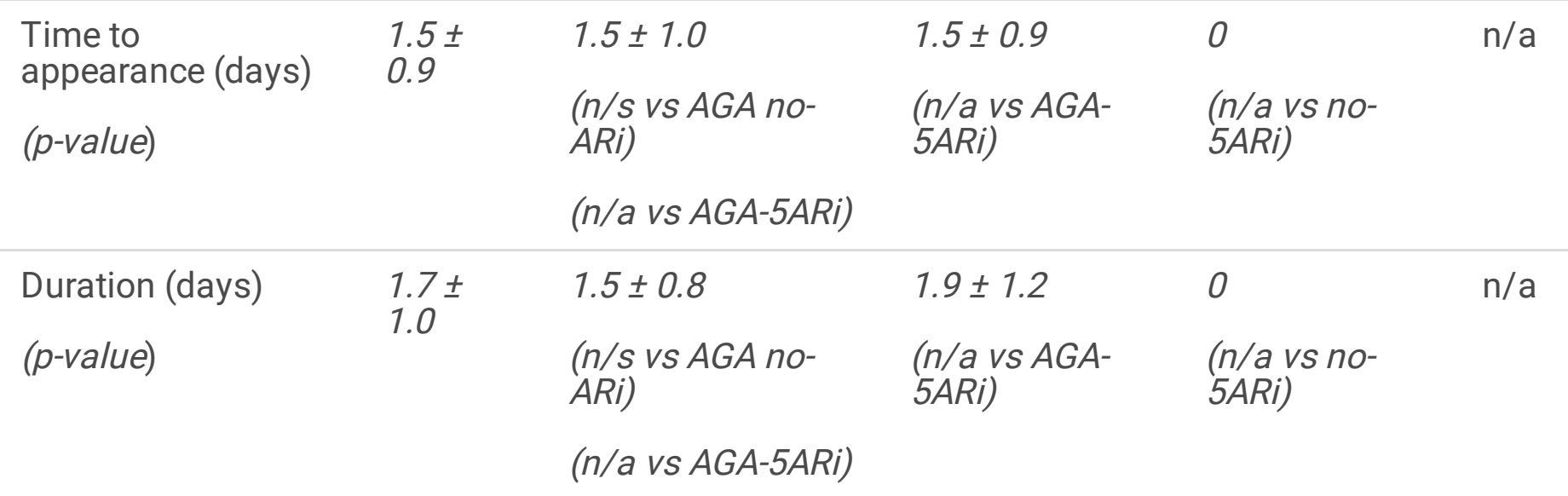

\section{Fatigue}

Presence (\%)
$45(23.4 \%)$

(35.2\%)
$41(57.7 \%)$

Page $30 / 57$
$<$

0.0001 


\begin{tabular}{|c|c|c|c|c|c|}
\hline (p-value) & & $\begin{array}{l}(p=0.006 \text { vs } A G A \\
\text { no-ARi) } \\
(p<0.0001 \text { vs } A G A- \\
5 A R i)\end{array}$ & $\begin{array}{l}(p<0.0001 \text { vs } \\
A G A-5 A R i)\end{array}$ & $\begin{array}{l}(p<0.0001 \text { vs } \\
\text { no-5ARi) }\end{array}$ & \\
\hline $\begin{array}{l}\text { Time to } \\
\text { appearance (days) } \\
\text { (p-value) }\end{array}$ & $\begin{array}{l}1.6 \pm \\
0.9\end{array}$ & $\begin{array}{l}1.6 \pm 0.9 \\
(\mathrm{n} / \mathrm{s} \text { vs } A G A \text { no- } \\
\text { ARi) } \\
\text { (n/a vs AGA-5ARi) }\end{array}$ & $\begin{array}{l}1.7 \pm 1.0 \\
(n / a \text { vs } A G A- \\
5 A R i)\end{array}$ & $\begin{array}{l}2 \\
(n / a \text { vs no- } \\
5 A R i)\end{array}$ & $\mathrm{n} / \mathrm{a}$ \\
\hline $\begin{array}{l}\text { Duration (days) } \\
\text { (p-value) }\end{array}$ & $\begin{array}{l}8.4 \pm \\
4.6\end{array}$ & $\begin{array}{l}7.6 \pm 3.8 \\
(p=0.033 \text { vs } A G A \\
\text { no-ARi) } \\
\text { (n/a vs AGA-5ARi) }\end{array}$ & $\begin{array}{l}9.8 \pm 5.6 \\
(n / a \text { vs } A G A- \\
5 A R i)\end{array}$ & $\begin{array}{l}6 \\
(n / a \text { vs no- } \\
5 A R i)\end{array}$ & $\mathrm{n} / \mathrm{a}$ \\
\hline Weakness & & & & & \\
\hline $\begin{array}{l}\text { Presence (\%) } \\
(p \text {-value) }\end{array}$ & $\begin{array}{l}92 \\
(29.5 \%)\end{array}$ & $\begin{array}{l}61(31.8 \%) \\
(n / s \text { vs } A G A \text { no- } \\
\text { ARi) } \\
\left(\begin{array}{l}(p<0.0001 \text { vs } A G A- \\
5 A R i)\end{array}\right.\end{array}$ & $\begin{array}{l}31(43.7 \%) \\
(p<0.0001 \text { vs } \\
A G A-5 A R i)\end{array}$ & $\begin{array}{l}1(1.9 \%) \\
(p<0.0001 \text { vs } \\
\text { no-5ARi) }\end{array}$ & $\begin{array}{l}< \\
0.0001\end{array}$ \\
\hline $\begin{array}{l}\text { Time to } \\
\text { appearance (days) } \\
\text { (p-value) }\end{array}$ & $\begin{array}{l}1.5 \pm \\
0.9\end{array}$ & $\begin{array}{l}1.5 \pm 0.8 \\
(\mathrm{n} / \mathrm{s} \text { vs } A G A \text { no- } \\
\text { ARi) } \\
\text { (n/a vs AGA-5ARi) }\end{array}$ & $\begin{array}{l}1.6 \pm 0.9 \\
(n / a \text { vs } A G A- \\
5 A R i)\end{array}$ & $\begin{array}{l}2 \\
(n / a \text { vs no- } \\
5 A R i)\end{array}$ & $\mathrm{n} / \mathrm{a}$ \\
\hline $\begin{array}{l}\text { Duration (days) } \\
\text { (p-value) }\end{array}$ & $\begin{array}{l}3.1 \pm \\
2.2\end{array}$ & $\begin{array}{l}2.8 \pm 1.7 \\
(p=0.031 \text { vs } A G A \\
\text { no-ARi) } \\
\text { (n/a vs AGA-5ARi) }\end{array}$ & $\begin{array}{l}4.0 \pm 2.8 \\
(n / a \text { vs } A G A- \\
5 A R i)\end{array}$ & $\begin{array}{l}1 \\
(n / a \text { vs no- } \\
5 A R i)\end{array}$ & $\mathrm{n} / \mathrm{a}$ \\
\hline Myalgia & & & & & \\
\hline $\begin{array}{l}\text { Presence (\%) } \\
\text { (p-value) }\end{array}$ & $\begin{array}{l}83 \\
(26.3 \%)\end{array}$ & $\begin{array}{l}53(27.6 \%) \\
(n / s(0.072)-v s \\
A G A \text { no-ARi) } \\
(p<0.0001 \text { vs } A G A- \\
5 A R i)\end{array}$ & $\begin{array}{l}30(42.3 \%) \\
(p<0.0001 \text { vs } \\
A G A-5 A R i)\end{array}$ & $\begin{array}{l}0 \\
(p<0.0001 \mathrm{vs} \\
\text { no-5ARi) }\end{array}$ & $\begin{array}{l}< \\
0.0001\end{array}$ \\
\hline $\begin{array}{l}\text { Time to } \\
\text { appearance (days) }\end{array}$ & $\begin{array}{l}1.6 \pm \\
0.9\end{array}$ & $1.6 \pm 0.8$ & $1.8 \pm 1.0$ & 0 & $\mathrm{n} / \mathrm{a}$ \\
\hline
\end{tabular}




\begin{tabular}{|c|c|c|c|c|}
\hline (p-value) & & $\begin{array}{l}(n / s \text { vs } A G A \text { no- } \\
\text { ARi) }\end{array}$ & $\begin{array}{l}\text { (n/a vs } A G A- \\
5 A R i)\end{array}$ & $\begin{array}{l}(n / a \text { vs no- } \\
5 A R i)\end{array}$ \\
\hline & & (n/a vs AGA-5ARi) & & \\
\hline Duration (days) & $3.6 \pm$ & $3.3 \pm 1.8$ & $4.1 \pm 1.6$ & 0 \\
\hline (p-value) & & $\begin{array}{l}(p=0.043 \text { vs } A G A \\
n o-A R i)\end{array}$ & $\begin{array}{l}\text { (n/a vs AGA- } \\
5 A R i)\end{array}$ & $\begin{array}{l}(n / a \text { vs no- } \\
\text { 5ARi) }\end{array}$ \\
\hline & & (n/a vs AGA-5ARi) & & \\
\hline
\end{tabular}

\section{Arthralgia}

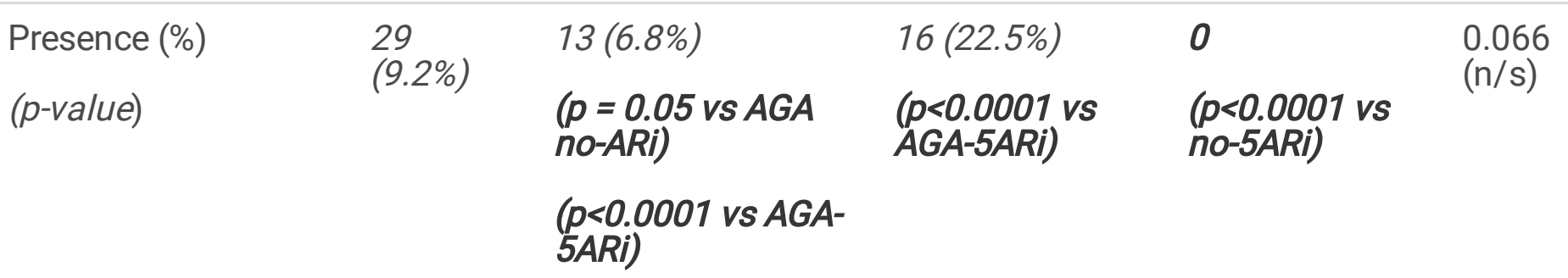

Time to

appearance (days)

(p-value)
$1.5 \pm 0.6$

(n/s vs AGA no-

ARi)

(n/a vs AGA-5ARi)
$2.4 \pm 0.9$

(n/s vs AGA no-

ARi)

(n/a vs AGA-5ARi)
$1.4 \pm 0.6$

(n/a vs AGA-

5ARi)

$2.6 \pm 1.4$

(n/a vs AGA-

5ARi)

(n)a vs no5ARi)

(p-value)
118

(37.5\%)

(p-value)
Time to

appearance (days)

(p-value)
$1.6 \pm$

0.9
$1.5 \pm 0.9$

(n/s vs AGA noARi)

(n/a vs AGA-5ARi)

Duration (days)

(p-value)
$4.3 \pm$

2.0
$4.0 \pm 1.7$ (n/a vs no5ARi) $\mathrm{n} / \mathrm{a}$

$88(45.8 \%)$

(n/s vs AGA no-

ARi)

( $p<0.0001$ vs AGA-

5ARi)
30 (42.3\%)

$(p<0.0001$ vs AGA-5ARi)
$<$ $(p<0.0001$ vs 0.0001 no-5ARi)

$1.6 \pm 0.9$

0 $\mathrm{n} / \mathrm{a}$

(n/a vs AGA5ARi)

(n/a vs no5ARi)

<

$5.1 \pm 2.3$

0

$\mathrm{n} / \mathrm{a}$ 
( $p=0.043$ vs $A G A$ no-ARi)

(n/a vs AGA-5ARi) (n/a vs AGA-

5ARi)

(n/a vs no-

5ARi)

\section{Thoracic pain}

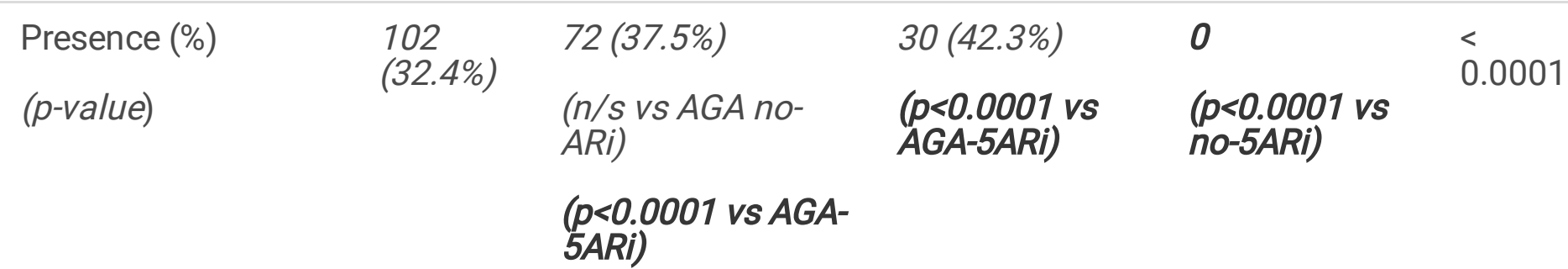

Time to

appearance (days)

(p-value)

$2.9 \pm \quad 2.8 \pm 1.2$

1.2

(n/s vs AGA no-

ARi)

(n/a vs AGA-5ARi)

Duration (days)

(p-value)
$4.3 \pm$

2.2
$4.1 \pm 2.1$

(n/s vs AGA no-

$4.8 \pm 2.3$

0

$\mathrm{n} / \mathrm{a}$

ARi)

(n/a vs AGA-

5ARi)

(n/a vs no5ARi)

(n/a vs AGA-5ARi)

\section{Upper back pain}

$\begin{array}{lll}\text { Presence (\%) } & 44 & 22(11.5 \%) \\ \text { (p-value) } & (14.0 \%) & (p=0.024 \text { vs } A G A \\ & & \text { no-ARi) } \\ & (p<0.0001 \text { vs } A G A- \\ & & 5 A R i)\end{array}$

Time to

appearance (days)

(p-value)

$2.0 \pm$

1.0

$2.0 \pm 0.9$

(n/s vs AGA no-

ARi)

(n/a vs AGA-5ARi)

Duration (days)

(p-value)
$4.3 \pm$

1.8
$4.3 \pm 1.6$

(n/s vs AGA noARi)
21 (29.6\%)

( $p<0.0001$ vs AGA-5ARi) $\mathrm{n} / \mathrm{a}$

(n/a vs no5ARi) 


\begin{tabular}{|c|c|c|c|c|c|}
\hline $\begin{array}{l}\text { Presence (\%) } \\
(p \text {-value })\end{array}$ & $\begin{array}{l}13 \\
(4.1 \%)\end{array}$ & $\begin{array}{l}5(2.6 \%) \\
(n / s \text { vs } A G A \text { no- } \\
A R i) \\
(p<0.0001 \text { vs } A G A- \\
5 A R i)\end{array}$ & $\begin{array}{l}8(11.3 \%) \\
(p<0.0001 \text { vs } \\
A G A-5 A R i)\end{array}$ & $\begin{array}{l}0 \\
(p<0.0001 \mathrm{vs} \\
\text { no-5ARi) }\end{array}$ & $\begin{array}{l}0.48 \\
(\mathrm{n} / \mathrm{s})\end{array}$ \\
\hline $\begin{array}{l}\text { Time to } \\
\text { appearance (days) } \\
\text { (p-value) }\end{array}$ & $\begin{array}{l}1.7 \pm \\
0.9\end{array}$ & $\begin{array}{l}2.0 \pm 1.1 \\
(p=0.046 \text { vs } A G A \\
\text { no-ARi) } \\
\text { (n/a vs AGA-5ARi) }\end{array}$ & $\begin{array}{l}1.5 \pm 0.7 \\
(n / a \text { vs } A G A- \\
5 A R i)\end{array}$ & $\begin{array}{l}0 \\
(n / a \text { vs no- } \\
5 A R i)\end{array}$ & $\mathrm{n} / \mathrm{a}$ \\
\hline $\begin{array}{l}\text { Duration (days) } \\
\text { (p-value) }\end{array}$ & $\begin{array}{l}3.9 \pm \\
1.5\end{array}$ & $\begin{array}{l}4.2 \pm 1.5 \\
(n / s \text { vs } A G A \text { no- } \\
\text { ARi) } \\
\text { (n/a vs AGA-5ARi) }\end{array}$ & $\begin{array}{l}3.7 \pm 1.5 \\
(n / a \text { vs } A G A- \\
5 A R i)\end{array}$ & $\begin{array}{l}0 \\
(n / a \text { vs no- } \\
5 A R i)\end{array}$ & $\mathrm{n} / \mathrm{a}$ \\
\hline Diarrhea & & & & & \\
\hline $\begin{array}{l}\text { Presence (\%) } \\
\text { (p-value) }\end{array}$ & $\begin{array}{l}66 \\
(20.9 \%)\end{array}$ & $\begin{array}{l}45(23.4 \%) \\
(n / s \text { vs } A G A \text { no- } \\
A R i) \\
(p=0.03 \text { vs } A G A- \\
5 A R i)\end{array}$ & $\begin{array}{l}19(26.8 \%) \\
(p=0.02 \text { vs } \\
A G A-5 A R i)\end{array}$ & $\begin{array}{l}2(3.8 \%) \\
(p=0.03 \text { vs no- } \\
5 A R i)\end{array}$ & $\begin{array}{l}0.06 \\
(\mathrm{n} / \mathrm{s})\end{array}$ \\
\hline $\begin{array}{l}\text { Time to } \\
\text { appearance (days) } \\
\text { (p-value) }\end{array}$ & $\begin{array}{l}1.9 \pm \\
1.1\end{array}$ & $\begin{array}{l}1.7 \pm 0.9 \\
(n / s \text { vs } A G A \text { no- } \\
\text { ARi) } \\
\text { (n/a vs AGA-5ARi) }\end{array}$ & $\begin{array}{l}2.4 \pm 1.4 \\
\left(n / a \text { vs } A G A^{-}\right. \\
5 A R i)\end{array}$ & $\begin{array}{l}1 \pm 0(1 ; 1) \\
(n / a \text { vs no- } \\
5 A R i)\end{array}$ & $\mathrm{n} / \mathrm{a}$ \\
\hline $\begin{array}{l}\text { Duration (days) } \\
\text { (p-value) }\end{array}$ & $\begin{array}{l}3.2 \pm \\
1.9\end{array}$ & $\begin{array}{l}2.9 \pm 1.6 \\
(n / s \text { vs } A G A \text { no- } \\
\text { ARi) } \\
\text { (n/a vs AGA-5ARi) }\end{array}$ & $\begin{array}{l}4.0 \pm 2.3 \\
(n / a \text { vs } A G A- \\
5 A R i)\end{array}$ & $\begin{array}{l}3 \pm 1(2 ; 4) \\
(n / a \text { vs no- } \\
5 A R i)\end{array}$ & $\mathrm{n} / \mathrm{a}$ \\
\hline Nauseas & & & & & \\
\hline $\begin{array}{l}\text { Presence (\%) } \\
(p-v a l u e)\end{array}$ & $\begin{array}{l}17 \\
(5.7 \%)\end{array}$ & $\begin{array}{l}10(5.2 \%) \\
(n / s \text { vs } A G A \text { no- } \\
\text { ARi) } \\
(n / s \text { vs } A G A-5 A R i)\end{array}$ & $\begin{array}{l}7(9.9 \%) \\
(n / s v s A G A- \\
5 A R i)\end{array}$ & $\begin{array}{l}0 \\
(n / s v s \text { no- } \\
5 A R i)\end{array}$ & $\begin{array}{l}0.55 \\
(\mathrm{n} / \mathrm{s})\end{array}$ \\
\hline
\end{tabular}




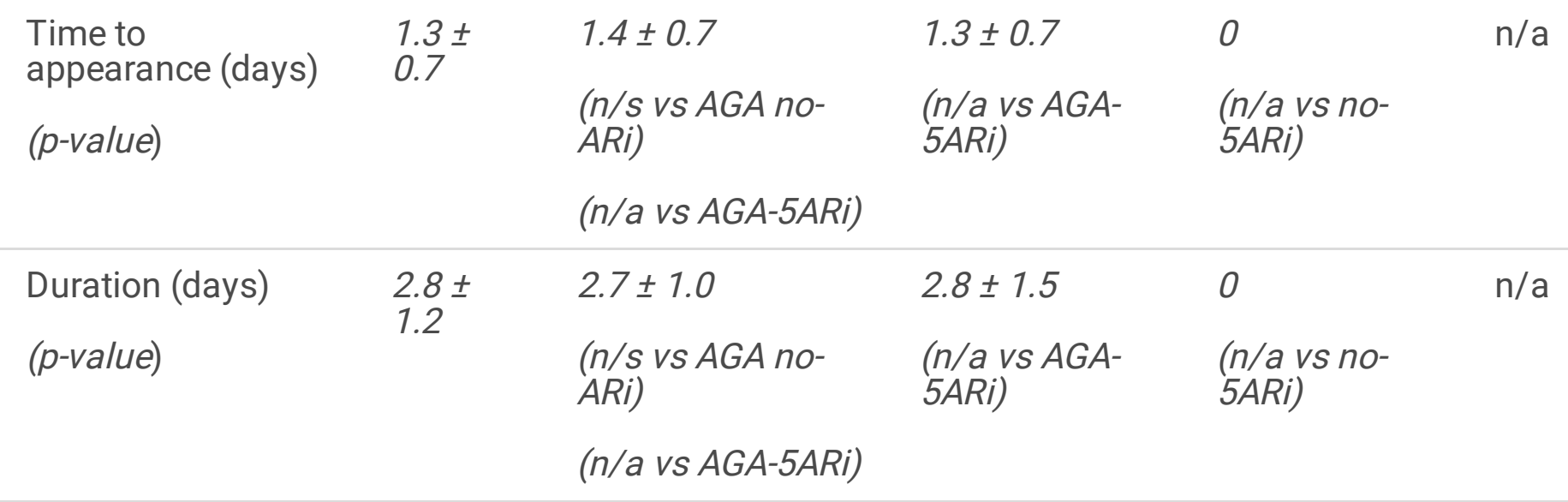

\section{Vomiting}

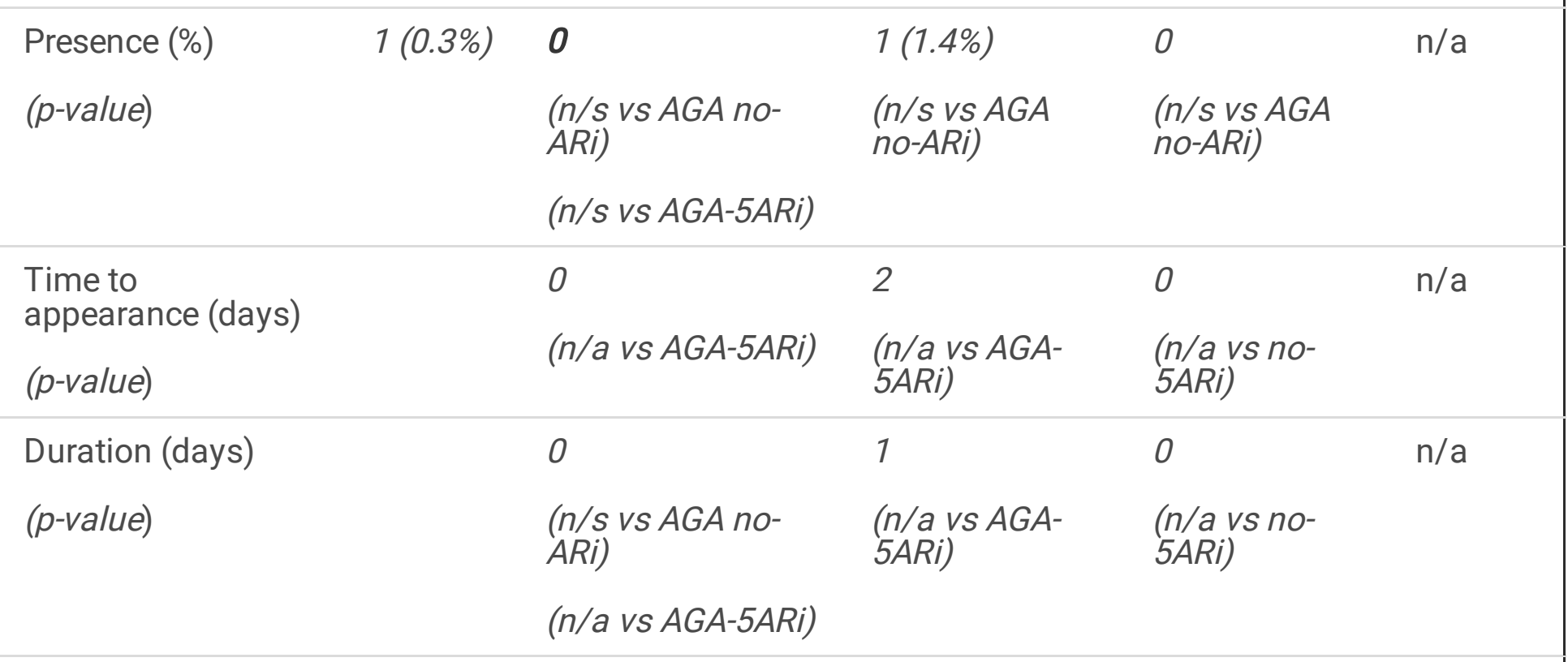

\section{Abdominal pain}

\begin{tabular}{|c|c|c|c|c|c|}
\hline $\begin{array}{l}\text { Presence (\%) } \\
(p-v a l u e)\end{array}$ & $\begin{array}{l}13 \\
(4.1 \%)\end{array}$ & $\begin{array}{l}7(3.6 \%) \\
(n / s \text { vs } A G A \text { no- } \\
A R i) \\
(n / s \text { vs } A G A-5 A R i)\end{array}$ & $\begin{array}{l}6(8.5 \%) \\
(n / s \text { vs AGA- } \\
5 A R i)\end{array}$ & $\begin{array}{l}0 \\
(n / s \text { vs } A G A- \\
5 A R i)\end{array}$ & 0.71 \\
\hline $\begin{array}{l}\text { Time to } \\
\text { appearance (days) } \\
\text { ( } p \text {-value) }\end{array}$ & $\begin{array}{l}1.4 \pm \\
0.7\end{array}$ & $\begin{array}{l}1.4 \pm 0.7 \\
(n / s \text { vs } A G A \text { no- } \\
A R i) \\
(n / a \text { vs } A G A-5 A R i)\end{array}$ & $\begin{array}{l}1.3 \pm 0.7 \\
(n / a \text { vs } A G A- \\
5 A R i)\end{array}$ & $\begin{array}{l}0 \\
(n / a \text { vs no- } \\
5 A R i)\end{array}$ & $\mathrm{n} / \mathrm{a}$ \\
\hline $\begin{array}{l}\text { Duration (days) } \\
\text { (p-value) }\end{array}$ & $\begin{array}{l}2.6 \pm \\
1.3\end{array}$ & $\begin{array}{l}2.0 \pm 0.9 \\
(\mathrm{n} / \mathrm{s} v s \text { sGA no- } \\
\text { ARi })\end{array}$ & $\begin{array}{l}3.3 \pm 1.2 \\
(n / a \text { vs } A G A- \\
5 A R i)\end{array}$ & $\begin{array}{l}0 \\
(n / a \text { vs no- } \\
5 A R i)\end{array}$ & $\mathrm{n} / \mathrm{a}$ \\
\hline
\end{tabular}




\section{Conjunctival \\ hyperemia}

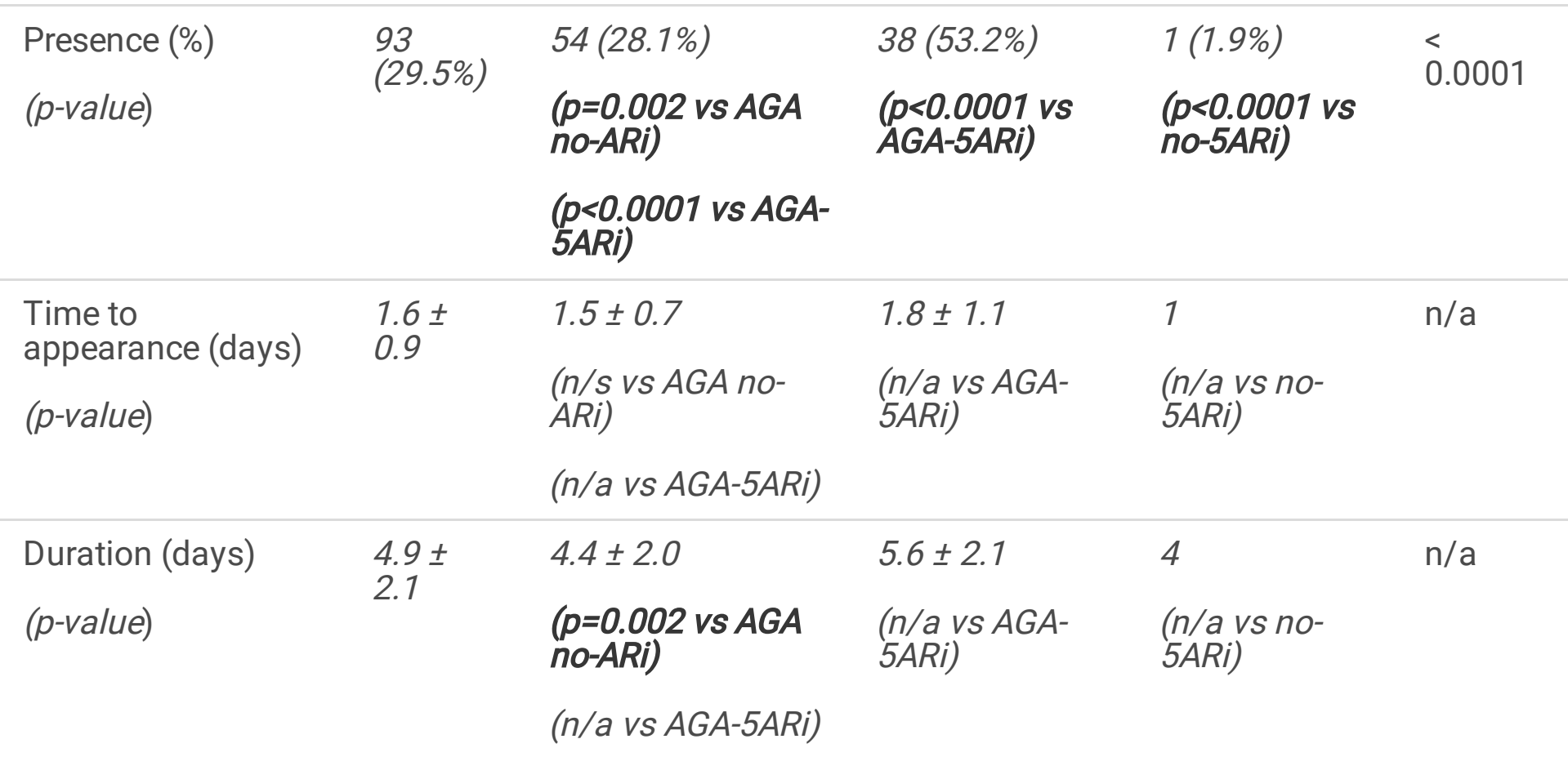

\section{Pre-orbital pain}

\begin{tabular}{|c|c|c|c|c|c|}
\hline $\begin{array}{l}\text { Presence (\%) } \\
\text { (p-value) }\end{array}$ & $\begin{array}{l}11 \\
(3.5 \%)\end{array}$ & $\begin{array}{l}8(4.2 \%) \\
(n / s \text { vs } A G A \text { no- } \\
A R i) \\
(n / s \text { vs AGA-5ARi) }\end{array}$ & $\begin{array}{l}2(2.8 \%) \\
(n / s \text { vs AGA- } \\
5 A R i)\end{array}$ & $\begin{array}{l}1(1.9 \%) \\
(n / s \text { vs no- } \\
5 A R i)\end{array}$ & $\begin{array}{l}0.96 \\
(\mathrm{n} / \mathrm{s})\end{array}$ \\
\hline $\begin{array}{l}\text { Time to } \\
\text { appearance (days) } \\
\text { (p-value) }\end{array}$ & $\begin{array}{l}1.3 \pm \\
0.6\end{array}$ & $\begin{array}{l}1.4 \pm 0.7 \\
(n / a \text { vs } A G A \text { no- } \\
A R i) \\
\text { (n/a vs AGA-5ARi) }\end{array}$ & $\begin{array}{l}1(1 ; 1) \\
(n / a \text { vs } A G A- \\
5 A R i)\end{array}$ & $\begin{array}{l}1 \\
(n / a \text { vs no- } \\
5 A R i)\end{array}$ & $\mathrm{n} / \mathrm{a}$ \\
\hline $\begin{array}{l}\text { Duration (days) } \\
\text { ( } p \text {-value) }\end{array}$ & $\begin{array}{l}3.6 \pm \\
1.6\end{array}$ & $\begin{array}{l}4.1 \pm 1.6 \\
(n / a \text { vs } A G A \text { no- } \\
\text { ARi) } \\
\text { (n/a vs AGA-5ARi) }\end{array}$ & $\begin{array}{l}2.5 \pm 0.5(2 ; 3) \\
(n / a \text { vs } A G A- \\
5 A R i)\end{array}$ & $\begin{array}{l}2 \\
(n / a \text { vs no- } \\
5 A R i)\end{array}$ & $\mathrm{n} / \mathrm{a}$ \\
\hline
\end{tabular}

No-5ARi = AGA no 5ARi + non-AGA groups; AGA = androgenetic alopecia; 5ARi = Androgen receptor inhibitors; $\mathrm{n} / \mathrm{s}=$ non-significant; $\mathrm{n} / \mathrm{a}=$ non-applicable 
Table 8. Drugs prescribed for COVID-19.

\begin{tabular}{|llllll|}
\hline $\begin{array}{l}\text { Medications } \\
\text { (+ azithromycin) }\end{array}$ & $\begin{array}{l}\text { Overall } \\
\text { males } \\
(\mathbf{n}=305)\end{array}$ & $\begin{array}{l}\text { Non-AGA } \\
\text { males } \\
(\mathbf{n}=192)\end{array}$ & $\begin{array}{l}\text { AGA no-5ARi } \\
\text { males } \\
(\mathbf{n}=71)\end{array}$ & $\begin{array}{l}\text { AGA-5ARi } \\
(\mathbf{n}=52)\end{array}$ & $\begin{array}{l}\text { p-value } \\
\text { (overall) }\end{array}$ \\
\hline Hydroxichloroquine & $68(21.6 \%)$ & $42(21.9 \%)$ & $10(14.1 \%)$ & $\begin{array}{l}16 \\
(30.8 \%)\end{array}$ & $\begin{array}{l}0.082 \\
(\mathrm{n} / \mathrm{s})\end{array}$ \\
\hline Nitazoxanide & $228(72.4 \%)$ & $141(74.4 \%) *$ & $58(81.7 \%)^{\#}$ & $\begin{array}{l}29 \\
(55.8 \%)^{\&}\end{array}$ & 0.0006 \\
\hline Ivermectin & $46(14.6 \%)$ & $30(15.6 \%)$ & $7(9.9 \%)$ & $9(17.3 \%)$ & $0.29(\mathrm{n} / \mathrm{s})$ \\
\hline Dutasteride & $\begin{array}{l}69 \\
(21.9 \% * *)\end{array}$ & $40(20.8 \%) *$ & $29(40.8 \%)$ & $n / a$ & 0.001 \\
\hline Spironolactone & $31(9.8 \% * *)$ & $19(9.9 \%)$ & $12(16.9 \%)$ & 0 & $0.17(\mathrm{n} / \mathrm{s})$ \\
\hline
\end{tabular}

$\mathrm{AGA}=$ androgenetic alopecia; $5 \mathrm{ARi}=$ Androgen receptor inhibitors; $\mathrm{n} / \mathrm{s}=$ non-significant; $\mathrm{n} / \mathrm{a}=$ nonapplicable

${ }^{*} \mathrm{p}<0.05$ vs AGA no-ARi males

${ }^{\mathrm{p}} \mathrm{p}<0.05$ vs AGA-ARi males

\& $p<0.05$ vs no-5ARi users (non-AGA and AGA no-5ARi)

**Not including AGA-5ARi users

Table 9. Additional drugs and supplements used to treat COVID-19. 


\begin{tabular}{|c|c|c|c|c|c|}
\hline $\begin{array}{l}\text { Additional drugs or } \\
\text { supplements }\end{array}$ & $\begin{array}{l}\text { Overall } \\
\text { males } \\
(n=305)\end{array}$ & $\begin{array}{l}\text { Non-AGA } \\
\text { males } \\
(n=192)\end{array}$ & $\begin{array}{l}\text { AGA no-5ARi } \\
\text { males } \\
(n=71)\end{array}$ & $\begin{array}{l}\text { AGA- } \\
\text { 5ARi } \\
(n=52)\end{array}$ & $\begin{array}{l}\text { p-value } \\
\text { (overall) }\end{array}$ \\
\hline Xa factor inhibitors & $34(10.8 \%)$ & $22(11.5 \%)$ & $9(12.7 \%)$ & $3(5.8 \%)$ & $\begin{array}{l}0.78 \\
(\mathrm{n} / \mathrm{s})\end{array}$ \\
\hline Enoxaparin & $19(6.0 \%)$ & $10(5.2 \%)$ & $9(12.7 \%)$ & 0 & $\begin{array}{l}0.46 \\
(n / s)\end{array}$ \\
\hline Glucocorticoids & $20(6.3 \%)$ & $11(5.7 \%)$ & $9(12.7 \%)$ & 0 & $\begin{array}{l}0.47 \\
(\mathrm{n} / \mathrm{s})\end{array}$ \\
\hline Vitamin C & $43(13.6 \%)$ & $16(22.5 \%)$ & $3(5.8 \%)$ & $3(5.8 \%)$ & $\begin{array}{l}0.26 \\
(\mathrm{n} / \mathrm{s})\end{array}$ \\
\hline Zinc & $46(14.6 \%)$ & $26(13.5 \%)$ & $16(22.5 \%)$ & $4(7.7 \%)$ & $\begin{array}{l}0.32 \\
(\mathrm{n} / \mathrm{s})\end{array}$ \\
\hline Vitamin D & $\begin{array}{l}268 \\
(88.2 \%)\end{array}$ & $174(90.6 \%)$ & 51 (85.9\%) & $\begin{array}{l}43 \\
(82.7 \%)\end{array}$ & $\begin{array}{l}0.63 \\
(\mathrm{n} / \mathrm{s})\end{array}$ \\
\hline
\end{tabular}

$\mathrm{AGA}=$ androgenetic alopecia; $5 \mathrm{ARi}=$ Androgen receptor inhibitors; $\mathrm{n} / \mathrm{s}=$ non-significant

COVID-19 course and outcomes

Tables 10 to 15 depict COVID-19 course and outcomes. Table 10 shows the average time-to-treat and the speed of COVID-19 resolution. Table 11 depicts the course of the clinical manifestations in COVID-19. Table 12 depicts the scores from the WHO COVID Ordinal Outcomes. Table 13 describes the mean loss of capacity for everyday activities. Table 14 describes the level of lung injury through radiology. Table 15 summarizes the rates of disease progression.

As shown in Table 10, time-to-treat was significantly lower in AGA-5ARi, compared to non-AGA and AGA no-5ARi males, which remained significant after adjustment for only symptomatic patients.

Duration of positive rtPCR-SARS-CoV-2, symptoms with or without anosmia and ageusia were significantly lower in 5ARi users compared to non-AGA, AGA no-5ARI, and overall non-users, and were lower in non-AGA compared to AGA no-5ARi males.

As depicted in Table 11, because $82.7 \%$ of 5ARi users remained asymptomatic throughout the COVID-19 infection, this group had significantly lower clinical manifestations compared to non-AGA, AGA no-5ARi and overall no-ARi users between seven days before until seven days after the beginning of treatment.

AGA no-5ARi had slower although not milder progression of symptoms, which may justify why this group was more affected since Days -7 to -4 . Non-AGA remained significantly less affected by COVID-19 
manifestations than AGA no-5ARi males between Days 0 and 7. AGA no-5ARi had similar speed of improvement compared to non-AGA.

Following the World Health Organization (WHO) COVID Ordinal Outcomes, AGA-5ARi group had lower scores than non-AGA, AGA no-5ARi, and overall no-5ARi users in Day 0. Conversely, at Day 7, AGA no-5ARi males had higher scores than non-AGA and AGA-5ARi groups, while these were similar between them.

From Day 14 to Day 60, groups became similar. In none of the days any patient progressed to Stages 3 to 5. Table 12 details clinical outcomes according to the WHO COVID Ordinal Outcomes.

In regards with the ability to perform everyday activities in an independent manner (Table 13), 5ARi users had lower loss of ability to perform everyday activities compared to non-AGA, AGA no-5ARi, and overall no-5ARi users, in Days 0 and 3, while these differences remitted from Day 7 to Day 30. Non-AGA males had lower loss of ability for everyday activities compared to AGA no-5ARi in Days 0 and 3. Overall, 5ARi users had no loss of ability to perform activities independently, while approximately $10 \%$ and $25 \%$ of the capacity was lost in average for non-AGA and AGA no-5ARi males, respectively.

Inability to perform everyday activities at Days 3, 7, 14 and 30 among AGA no-5ARi males were similar to Days $0,3,7$ and 14 in non-AGA males, respectively. It means that the average loss of ability was similar between Day 3 in AGA no-5ARi males and Day 0 in non-AGA males, between Day 7 in AGA no-5ARi males and Day 3 in non-AGA males, between Day 14 in AGA no-5ARi males and Day 7 in non-AGA males, and between Day 30 in AGA no-5ARi males and Day 14 in non-AGA males.

In terms of chest computerized tomography (CT) scan (Table 14), AGA-5ARi males had significantly less compromised lungs compared to non-AGA, AGA no-5ARi, and overall males, in Days 0 and 7 (Days 14 and 30 had insufficient number of patients for comparison purposes). Non-AGA males had less affected lungs compared to AGA no-5ARi at all times (Table 14).

In terms of radiological progression, of 116 that underwent at least two CT scans, one (0.9\%) had progression of lung lesion from the first to the second CT scan. More important improvement was observed between Days 0 and 7 for 5ARi users, between Days 7 and 14 for non-AGA males, and between Days 14 and 21 for AGA no-5ARi males.

As shown in Table 15, all 305 patients remained in zero score in the Brescia Respiratory Severity Scale, and none of the patients required hospitalization, mechanical ventilation, or need of noradrenaline or dopamine, or died.

Table 10. COVID-19 clinical outcomes. 


\begin{tabular}{|c|c|c|c|c|c|}
\hline $\begin{array}{l}\text { Clinical } \\
\text { clustering }\end{array}$ & $\begin{array}{l}\text { Overall } \\
\text { males } \\
(n= \\
(n 05)\end{array}$ & $\begin{array}{l}\text { Non-AGA } \\
\text { males } \\
(n=192)\end{array}$ & $\begin{array}{l}\text { AGA no- } \\
5 \text { ARi } \\
\text { males } \\
(n=71)\end{array}$ & $\begin{array}{l}\text { AGA-5ARi } \\
(n=52)\end{array}$ & $\begin{array}{l}\text { p-value } \\
\text { (overall) }\end{array}$ \\
\hline $\begin{array}{l}\text { Time-to-treat } \\
\text { (p-value) }\end{array}$ & $\begin{array}{l}2.8 \pm \\
1.8\end{array}$ & $\begin{array}{l}3.0 \pm 1.6 \\
(p<0.0001 \\
\text { vs AGA no- } \\
\text { ARi) } \\
(p<0.0001 \\
\text { vs AGA- } \\
5 A R i)\end{array}$ & $\begin{array}{l}4.0 \pm 1.3 \\
(p<0.0001 \\
\text { vs AGA- } \\
5 A R i)\end{array}$ & $\begin{array}{l}0.4 \pm 1.1 \text { (Median }=0 ; 95 \% \mathrm{Cl}= \\
0.3 \text { ) } \\
(p<0.0001 \text { vs no-5ARi) } \\
\text { ( } p<0.001 \text { vs no-5ARi after } \\
\text { adjustment for only } \\
\text { symptomatic patients) }\end{array}$ & $<.0001$ \\
\hline $\begin{array}{l}\text { Duration of } \\
\text { positive rtPCR } \\
\text { (days) } \\
\text { (p-value) }\end{array}$ & $\begin{array}{l}13.9 \pm \\
5.9\end{array}$ & $\begin{array}{l}14.0 \pm 5.16 \\
(p<0.0001 \\
\text { Vs AGA no- } \\
\text { ARi) } \\
(p<0.0001 \\
\text { vs AGA- } \\
5 A R i)\end{array}$ & $\begin{array}{l}17.8 \pm 6.2 \\
(p<0.0001 \\
\text { vs AGA- } \\
5 A R i)\end{array}$ & $\begin{array}{l}8.2 \pm 3.0 \\
\text { ( } p<0.0001 \text { vs no-5ARi) } \\
\text { ( } p=0.002 \text { vs no-5ARi after } \\
\text { adjustment for only } \\
\text { symptomatic patients) }\end{array}$ & $\begin{array}{l}<.0001 \\
0 .\end{array}$ \\
\hline $\begin{array}{l}\text { Remission not } \\
\text { including } \\
\text { anosmia (days) } \\
\text { (without ( } p \text { - } \\
\text { value) }\end{array}$ & $\begin{array}{l}5.7 \pm \\
4.5\end{array}$ & $\begin{array}{l}5.9 \pm 3.7 \\
\text { p<0.0001 } \\
\text { Vs AGA no- } \\
\text { ARi) } \\
(p<0.0001 \\
\text { vs AGA- } \\
5 A R i)\end{array}$ & $\begin{array}{l}8.8 \pm 4.7 \\
(p<0.0001 \\
\text { Vs AGA- } \\
5 A R i)\end{array}$ & $\begin{array}{l}0.7 \pm 1.6 \text { (Median }=0 ; 95 \% \mathrm{Cl}= \\
0.4) \\
(p<0.0001 \text { vs no-5ARi) } \\
\text { ( } p<0.0001 \text { vs no-5ARi after } \\
\text { adjustment for only } \\
\text { symptomatic patients) }\end{array}$ & $\begin{array}{l}<.0001 \\
0 .\end{array}$ \\
\hline $\begin{array}{l}\text { Remission } \\
\text { including } \\
\text { anosmia (days) } \\
\text { (without ( } p \text { - } \\
\text { value) }\end{array}$ & $\begin{array}{l}9.1 \pm \\
7.2\end{array}$ & $\begin{array}{l}9.4 \pm 6.0 \\
(p<0.0001 \\
\text { Vs AGA no- } \\
\text { ARi) } \\
(p<0.0001 \\
\text { vs AGA- } \\
5 A R i)\end{array}$ & $\begin{array}{l}14.2 \pm 7.3 \\
(p<0.0001 \\
\text { vs AGA- } \\
5 A R i)\end{array}$ & $\begin{array}{l}0.9 \pm 2.0 \text { (Median }=0 ; 95 \% \mathrm{Cl}= \\
0.6) \\
(p<0.0001 \text { vs no-5ARi) } \\
\text { ( } p<0.001 \text { vs no-5ARi after } \\
\text { adjustment for only } \\
\text { symptomatic patients) }\end{array}$ & $<.0001$ \\
\hline
\end{tabular}

Table 11. COVID-19 course. 


\begin{tabular}{|c|c|c|c|c|c|}
\hline $\begin{array}{l}\text { Clinical } \\
\text { evolution }\end{array}$ & $\begin{array}{l}\text { Overall males } \\
(n=305)\end{array}$ & $\begin{array}{l}\text { Non-AGA } \\
\text { males } \\
(n=192)\end{array}$ & $\begin{array}{l}\text { AGA no-5ARi } \\
\text { males } \\
(n=71)\end{array}$ & $\begin{array}{l}\text { AGA-5ARi } \\
(n=52)\end{array}$ & $\begin{array}{l}\text { p-value } \\
\text { (overall) }\end{array}$ \\
\hline $\begin{array}{l}\text { Days }-7 \\
\text { to }-4 \\
\text { (p-value) }\end{array}$ & $\begin{array}{l}88.2 \pm 29.7 \\
(\text { Median = } 100 ; \\
95 \% \mathrm{Cl}=3.3) \\
\text { (84.1\% } \\
\text { asymptomatic) }\end{array}$ & $\begin{array}{l}88.8 \pm 29.3 \\
(\text { Median = } 100 ; \\
95 \% \mathrm{Cl}=4.1) \\
{[165(85.9 \%)} \\
\text { asymptomatic] } \\
(p=0.03) \text { vs } \\
\text { AGA no-ARi) } \\
(\mathrm{n} / \mathrm{s} \text { vs AGA- } \\
5 A R i)\end{array}$ & $\begin{array}{l}78.1 \pm 37.1 \\
\text { (Median = 100; } \\
95 \% \mathrm{Cl}=8.6) \\
\text { [48 }(67.6 \%) \\
\text { asymptomatic] } \\
(p=0.002 \mathrm{vs} \\
\text { AGA-5ARi) }\end{array}$ & $\begin{array}{l}100.0 \pm 0 \\
(\text { Median }=100 \\
95 \% \mathrm{Cl}=0)[52 \\
(100 \%) \\
\text { asymptomatic }] \\
(p=0.03 \text { vs no- } \\
5 \text { ARi) }\end{array}$ & 0.008 \\
\hline $\begin{array}{l}\text { Days }-3 \\
\text { to }-1 \\
\text { (p-value) }\end{array}$ & $\begin{array}{l}39.6 \pm 42.7 \\
(\text { Median = } 10 ; \\
95 \% \mathrm{Cl}=4.7) \\
\text { (30.5\% } \\
\text { asymptomatic) }\end{array}$ & $\begin{array}{l}34.4 \pm 40.5 \\
(\text { Median }=10 ; \\
95 \% \mathrm{Cl}=5.7) \\
{[46(24.0 \%)} \\
\text { asymptomatic] } \\
(\mathrm{n} / \mathrm{s} \text { vs } A G A \text { no- } \\
\text { ARi) } \\
(p<0.0001 \text { vs } \\
\text { AGA-5ARi) }\end{array}$ & $\begin{array}{l}20.4 \pm 28.0 \\
(\text { Median }=10 ; \\
95 \% \mathrm{Cl}=6.5)^{[}[6 \\
(8.4 \%) \\
\text { asymptomatic] } \\
(p<0.0001 \text { vs } \\
\text { AGA-5ARi) }\end{array}$ & $\begin{array}{l}85.0 \pm 35.2 \\
\text { (Median = 100; } \\
95 \% \mathrm{Cl}=9.6)[44 \\
\text { (84.6\%) } \\
\text { asymptomatic } \\
\text { (p<0.0001 vs no- } \\
\text { 5ARi) }\end{array}$ & $\begin{array}{l}< \\
0.0001\end{array}$ \\
\hline $\begin{array}{l}\text { Day } 0 \\
\text { (p-value) }\end{array}$ & $\begin{array}{l}32.7 \pm 38.9 \\
(\text { Median }=10 ; \\
95 \% \mathrm{Cl}=4.3) \\
(18.3 \% \\
\text { asymptomatic) }\end{array}$ & $\begin{array}{l}24.5 \pm 33.8 \\
\text { (Median = } 10 ; \\
95 \% \mathrm{Cl}=4.8) \\
{[16(8.3 \%)} \\
\text { asymptomatic] } \\
(\mathrm{n} / \mathrm{s} \text { vs } A G A \text { no- } \\
\text { ARi) } \\
(p<0.0001 \text { vs } \\
\text { AGA-5ARi) }\end{array}$ & $\begin{array}{l}15.1 \pm 14.4 \\
(\mathrm{Median}=10 \\
95 \% \mathrm{Cl}=3.3)^{\prime}[0 \\
(0 \%) \\
\text { asymptomatic }] \\
(\mathbf{p}<0.0001 \text { vs } \\
\text { AGA-5ARi) }\end{array}$ & $\begin{array}{l}87.1 \pm 31.9 \\
(\text { Median = } 100 ; \\
95 \% \mathrm{Cl}=8.7)[43 \\
\text { (82.7\%) } \\
\text { asymptomatic] } \\
\text { (p<0.0001 vs no- } \\
5 \text { ARi) }\end{array}$ & $\begin{array}{l}< \\
0.0001\end{array}$ \\
\hline $\begin{array}{l}\text { Day } 1 \\
\text { (p-value) }\end{array}$ & $\begin{array}{l}52.7 \pm 36.3 \\
(\text { Median }=50 ; \\
95 \% \mathrm{Cl}=4.0) \\
(19.4 \% \\
\text { asymptomatic) }\end{array}$ & $\begin{array}{l}48.8 \pm 34.6 \\
(\text { Median = 45; } \\
95 \% \mathrm{Cl}=4.9) \\
{[17(8.9 \%)} \\
\text { asymptomatic] } \\
(p=0.021 \text { vs } \\
\text { AGA no-ARi) } \\
(p<0.0001 \text { vs } \\
\text { AGA-5ARi) }\end{array}$ & $\begin{array}{l}36.6 \pm 26.5 \\
(\text { Median = } 40 ; \\
95 \% \mathrm{Cl}=6.2)^{[} 11 \\
(1.4 \%) \\
\text { asymptomatic } \\
(P<0.0001 \text { vs } \\
\text { AGA-5ARi) }\end{array}$ & $\begin{array}{l}88.8 \pm 29.8 \\
\text { (Median = 100; } \\
95 \% \mathrm{Cl}=8.1)[43 \\
\text { (82.7\%) } \\
\text { asymptomatic] } \\
\text { (p<0.0001 vs no- } \\
\text { 5ARi) }\end{array}$ & $\begin{array}{l}<.0001 \\
0.00\end{array}$ \\
\hline $\begin{array}{l}\text { Day } 2 \\
\text { (p-value) }\end{array}$ & $\begin{array}{l}71.3 \pm 31.2 \\
(\text { Median = } 90 ; \\
95 \% \mathrm{Cl}=3.4) \\
(25.4 \% \\
\text { asymptomatic) }\end{array}$ & $\begin{array}{l}67.7 \pm 32.4 \\
(\text { Median = 90; } \\
95 \% \mathrm{Cl}=4.6) \\
{[29(15.1 \%)} \\
\text { asymptomatic } \\
(p=0.032 \text { vs } \\
\text { AGA no-ARi) }\end{array}$ & $\begin{array}{l}61.9 \pm 26.9 \\
(\text { Median = } 60 ; \\
95 \% \mathrm{Cl}=6.3)^{[}[4 \\
(5.6 \%) \\
\text { asymptomatic } \\
(p<0.0001 \text { vs } \\
\text { AGA-5ARi) }\end{array}$ & $\begin{array}{l}97.3 \pm 6.9 \\
(\text { Median = 100; } \\
95 \% \mathrm{Cl}=3.8)[47 \\
(90.4 \%) \\
\text { asymptomatic] } \\
\text { (p<0.0001 vs no- } \\
5 \text { ARi) }\end{array}$ & $\begin{array}{l}<.0001 \\
0.00\end{array}$ \\
\hline
\end{tabular}


(p<0.0001 vs

AGA-5ARi)

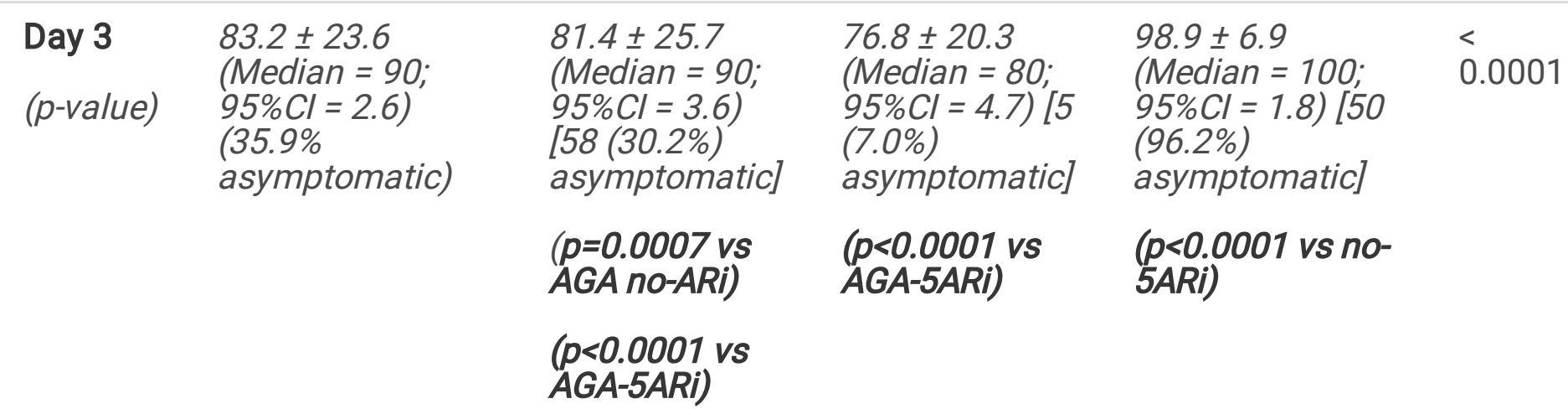

$\begin{array}{ll}\text { Day } 7 & 95.5 \pm 10.5 \\ & \text { (Median =100; } \\ \text { (p-value) } & 95 \% \mathrm{Cl}=1.2) \\ & (72.7 \% \\ & \text { asymptomatic) }\end{array}$

$95.7+10.9$

(Median = 100;

$91.5 \pm 11.6$

$100.0 \pm 0$

(Median = 100;

$<$

$95 \% \mathrm{Cl}=1.5$ )

[143 (74.5\%)

(Median $=95$;

$95 \% \mathrm{Cl}=0$ ) $[52$

$95 \% \mathrm{Cl}=2.7)$

(100\%)

asymptomatic]

asymptomatic]

asymptomatic]

$(p=0.0004$ vs

AGA no-ARi)

( $p=0.005$ vs

(p<0.0001 vs

AGA-5ARi)

$(p=0.0002$ vs no-

5ARi)

AGA-5ARi)

$\begin{array}{ll}\text { Day 14 } & 99.0 \pm 4.2 \\ \text { (p-value) } & (\text { Median = 100; } \\ & 95 \% \mathrm{Cl}=0.5) \\ & (92.4 \% \\ & \text { asymptomatic) }\end{array}$

$99.1 \pm 4.5$

(Median = 100;

$95 \% \mathrm{Cl}=0.6)$

[181 (94.3\%)

asymptomatic]

$98.0 \pm 4.8$

(Median = 100;

$95 \% \mathrm{Cl}=1.1)$

[58 (81.7\%)

(n/s vs AGA no-

ARi)

asymptomatic]

$100.0 \pm 0$

0.17

(Median = 100;

$95 \% \mathrm{Cl}=0$ ) $[52$

(n/s)

(100\%)

asymptomatic]

(n/s vs AGA-

5ARi)

$(n / s(p=0.083) v s$ no-5ARi)

(n/s vs AGA-

5ARi)

$\begin{array}{ll}\text { Day } 21 & 99.8 \pm 1.1 \\ & (\text { Median = 100 } \\ \text { (p-value) } & 95 \% \mathrm{Cl}=0.1) \\ & (62.8 \% \\ & \text { asymptomatic) }\end{array}$

$99.8 \pm 1.2$

(Median $=100$;

$95 \% \mathrm{Cl}=0.2)$

[187 (97.4\%)

asymptomatic]

(n/s vs AGA no-

ARi)

$99.6 \pm 1.3$

(Median = 100;

$95 \% \mathrm{Cl}=0.3)$

[66 (93.0\%)

asymptomatic]

$100.0 \pm 0$

1.0

(Median = 100;

$(\mathrm{n} / \mathrm{s})$

$95 \% \mathrm{Cl}=0)$ [52

(100\%)

asymptomatic]

(n/s vs AGA-

5ARi)

(n/s vs no-5ARi)

(n/s vs AGA-

5ARi)

$\begin{array}{ll}\text { Day 30 } & 99.9 \pm 0.5 \\ \text { ( } p \text {-value }) & 95 \% \mathrm{Cl}=0.1)[190\end{array}$
$95 \% \mathrm{Cl}=0.1)[190$

(99.0\%)

asymptomatic]
$99.9 \pm 0.5$

(Median = 100;

$95 \% \mathrm{Cl}=0.1)$

[190 (99.0\%)

asymptomatic]

(n/s vs AGA no-

ARi)
$99.9 \pm 0.8$

(Median = 100;

$95 \% \mathrm{Cl}=0.2)$

[69 (97.2\%)

asymptomatic]

( $n / s$ vs AGA-

5ARi)
$100.0 \pm 0$

(Median = 100;

$95 \% \mathrm{Cl}=0)[52$

(100\%)

asymptomatic]

(n/s vs no-5ARi)
1.0

$(\mathrm{n} / \mathrm{s})$ 
(n/s vs $A G A-$

5ARi)

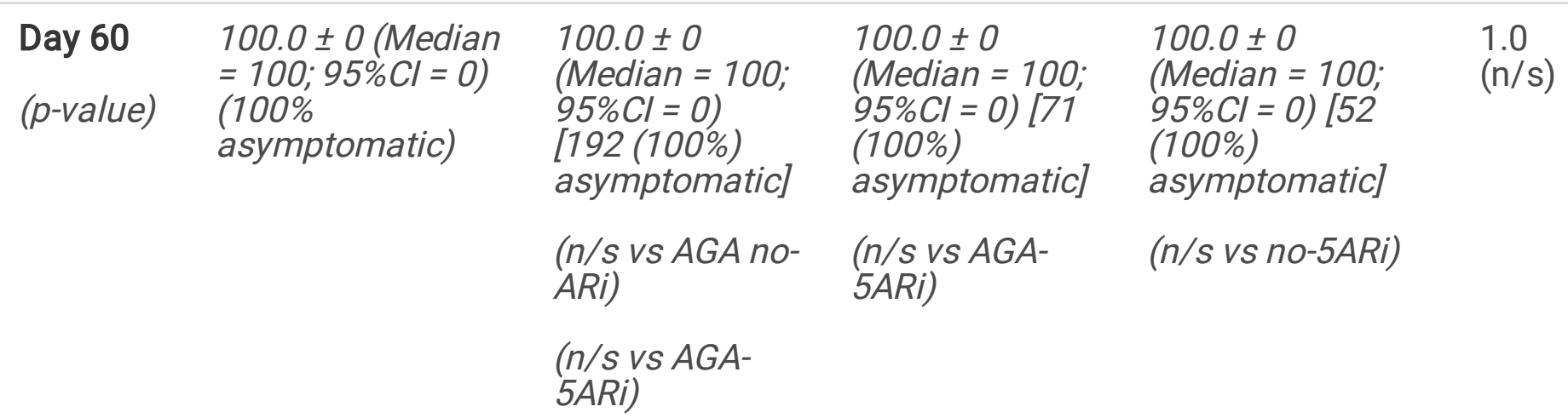

$\mathrm{AGA}=$ androgenetic alopecia; $5 \mathrm{ARi}=$ Androgen receptor inhibitors; $\mathrm{n} / \mathrm{s}=$ non-significant; $\mathrm{Cl}=$ confidence interval

Table 12. WHO COVID-19 Ordinal Outcomes. 


\begin{tabular}{|c|c|c|c|c|c|}
\hline $\begin{array}{l}\text { WHO COVID Ordinal } \\
\text { Outcomes }\end{array}$ & $\begin{array}{l}\text { Overall } \\
\text { males } \\
(n= \\
305)\end{array}$ & $\begin{array}{l}\text { Non-AGA males } \\
(n=192)\end{array}$ & $\begin{array}{l}\text { AGA no-5ARi } \\
\text { males } \\
(n=71)\end{array}$ & $\begin{array}{l}\text { AGA-5ARi } \\
(n=52)\end{array}$ & $\begin{array}{l}\text { p-value } \\
\text { (overall) }\end{array}$ \\
\hline $\begin{array}{l}\text { Day } 0 \\
\text { (p-value) }\end{array}$ & & $\begin{array}{l}(p<0.0001 \text { vs } \\
\text { AGA no-ARi) } \\
(p=0.0003 \text { vs } \\
\text { AGA-5ARi) }\end{array}$ & $\begin{array}{l}(p<0.0001 \text { vs } \\
A G A-5 A R i)\end{array}$ & $\begin{array}{l}(p<0.0001 \text { vs } \\
\text { no-5ARi) }\end{array}$ & $\begin{array}{l}< \\
0.0001\end{array}$ \\
\hline Stage 1 & $\begin{array}{l}199 \\
(43.2 \%)\end{array}$ & 125 (65.1\%) & $23(33.4 \%)$ & 51 (98.0\%) & \\
\hline Stage 2 & $\begin{array}{l}116 \\
(36.8 \%)\end{array}$ & $67(34.9 \%)$ & $48(67.6 \%)$ & 1 (2.0\%) & \\
\hline Stages 3-5 & 0 & 0 & 0 & 0 & \\
\hline $\begin{array}{l}\text { Day } 7 \\
\text { (p-value) }\end{array}$ & & $\begin{array}{l}(p=0.021 \text { vs } A G A \\
\text { no-ARi) } \\
(n / s \text { vs } A G A- \\
5 A R i)\end{array}$ & $\begin{array}{l}(p=0.015 \mathrm{vs} \\
A G A-5 A R i)\end{array}$ & $\begin{array}{l}(\mathrm{n} / \mathrm{s} \text { vs no- } \\
5 A R i)\end{array}$ & 0.021 \\
\hline Stage 1 & $\begin{array}{l}289 \\
(91.7 \%)\end{array}$ & $180(93.8 \%)$ & $57(80.3 \%)$ & $52(100 \%)$ & \\
\hline Stage 2 & $\begin{array}{l}26 \\
(8.3 \%)\end{array}$ & $12(6.2 \%)$ & $14(19.7 \%)$ & 0 & \\
\hline Stages 3-5 & 0 & 0 & 0 & 0 & \\
\hline $\begin{array}{l}\text { vs Day } 0 \\
\text { ( } p \text {-value) }\end{array}$ & $<0.0001$ & $<0.0001$ & $<0.0001$ & $1.0(\mathrm{n} / \mathrm{s})$ & \\
\hline $\begin{array}{l}\text { Day } 14 \\
\text { (p-value) }\end{array}$ & & $\begin{array}{l}(\mathrm{n} / \mathrm{s} \text { vs } A G A \text { no- } \\
\text { ARi) } \\
\\
(\mathrm{n} / \mathrm{s} \text { vs } A G A- \\
5 A R i)\end{array}$ & $\begin{array}{l}(n / s \text { vs } A G A- \\
5 A R i)\end{array}$ & $\begin{array}{l}(\mathrm{n} / \mathrm{s} \text { vs no- } \\
5 A R i)\end{array}$ & 0.90 \\
\hline Stage 1 & $\begin{array}{l}310 \\
(98.4 \%)\end{array}$ & $190(99.0 \%)$ & 68 (95.8\%) & 52 (100\%) & \\
\hline Stage 2 & $5(1.6 \%)$ & $2(1.0 \%)$ & $3(4.2 \%)$ & 0 & \\
\hline Stages 3-5 & 0 & 0 & 0 & 0 & \\
\hline
\end{tabular}




\begin{tabular}{|c|c|c|c|c|c|}
\hline $\begin{array}{l}\text { vs Day } 0 \\
\text { ( } p \text {-value) }\end{array}$ & $<0.0001$ & $<0.0001$ & $<0.0001$ & $1.0(n / s)$ & \\
\hline $\begin{array}{l}\text { vs Day } 7 \\
\text { ( } p \text {-value) }\end{array}$ & $\begin{array}{l}0.17 \\
(n / s)\end{array}$ & $0.38(\mathrm{n} / \mathrm{s})$ & $0.11(n / s)$ & $1.0(n / s)$ & \\
\hline $\begin{array}{l}\text { Days } 30 \text { and } 60 \\
\text { (p-value) }\end{array}$ & & $\begin{array}{l}(n / s \text { vs AGA no- } \\
\text { ARi) } \\
(n / s \text { vs AGA- } \\
5 A R i)\end{array}$ & $\begin{array}{l}(n / s \text { vs } A G A- \\
5 A R i)\end{array}$ & $\begin{array}{l}(n / s \text { vs no- } \\
\text { 5ARi) }\end{array}$ & \\
\hline Stage 1 & $\begin{array}{l}305 \\
(100 \%)\end{array}$ & $192(100 \%)$ & 71 (100\%) & $52(100 \%)$ & $\mathrm{n} / \mathrm{a}$ \\
\hline Stage 2 & 0 & 0 & 0 & 0 & $\mathrm{n} / \mathrm{a}$ \\
\hline Stages 3-5 & 0 & 0 & 0 & 0 & $\mathrm{n} / \mathrm{a}$ \\
\hline
\end{tabular}

$\mathrm{AGA}=$ androgenetic alopecia; $5 \mathrm{ARi}=$ Androgen receptor inhibitors; $\mathrm{n} / \mathrm{s}=$ non-significant; $\mathrm{Cl}$ = confidence interval

Table 13. Loss of ability to perform everyday activities due to COVID-19. 


\begin{tabular}{|c|c|c|c|c|c|}
\hline $\begin{array}{l}\text { Loss of } \\
\text { ability of } \\
\text { everyday } \\
\text { activities } \\
\text { (\%) }\end{array}$ & $\begin{array}{l}\text { Overall males } \\
(n=305)\end{array}$ & $\begin{array}{l}\text { Non-AGA males } \\
(n=192)\end{array}$ & $\begin{array}{l}\text { AGA no-5ARi } \\
\text { males } \\
(n=71)\end{array}$ & $\begin{array}{l}\text { AGA-5ARi } \\
(n=52)\end{array}$ & $\begin{array}{l}\text { p-value } \\
\text { (overall) }\end{array}$ \\
\hline $\begin{array}{l}\text { Day } 0 \\
\text { (p-value) }\end{array}$ & $\begin{array}{l}12.4 \pm 19.3 \\
\text { (Median = } 0 \\
95 \% \mathrm{Cl}=2.1) \\
\text { (63.2\% full } \\
\text { functional } \\
\text { capacity) }\end{array}$ & $\begin{array}{l}10.8 \pm 17.2 \\
\text { (Median = } \\
95 \% \mathrm{Cl}=2.4) \\
(65.1 \%) \text { full } \\
\text { functional } \\
\text { capacity] } \\
(p<0.0001 \text { vs } \\
\text { AGA no-ARi) } \\
(p=0.0002 \text { vs } \\
\text { AGA-5ARi) }\end{array}$ & $\begin{array}{l}25.6 \pm 23.4 \\
(\text { Median }=20 ; \\
95 \% C l=4.2) \\
(32.4 \%) \text { full } \\
\text { functional } \\
\text { capacity] } \\
(p<0.0001 \text { vs } \\
\text { AGA-5ARi) }\end{array}$ & $\begin{array}{l}0.4 \pm 2.7 \text { (Median } \\
=0 ; 95 \% \mathrm{Cl}=0.7) \\
{[51(98.1 \%) \text { full }} \\
\text { functional } \\
\text { capacity] } \\
(p<0.0001 \text { vs no- } \\
5 \text { ARi) }\end{array}$ & $\begin{array}{l}< \\
0.0001\end{array}$ \\
\hline $\begin{array}{l}\text { Day } 3 \\
\text { (p-value) }\end{array}$ & $\begin{array}{l}5.1 \pm 12.0 \\
\text { (Median = } 0 \\
95 \% \mathrm{Cl}=1.3 \text { ) } \\
\text { (78.4\% full } \\
\text { functional } \\
\text { capacity) }\end{array}$ & $\begin{array}{l}4.1 \pm 9.7 \text { (Median } \\
=0 ; 95 \% \mathrm{Cl}=1.4) \\
{[154(80.2 \%) \text { full }} \\
\text { functional } \\
\text { capacity] } \\
(p=0.0038 \text { vs } \\
\text { AGA no-ARi) } \\
(p=0.029 \text { vs } A G A- \\
5 A R i)\end{array}$ & $\begin{array}{l}11.7 \pm 18.0 \\
(\text { Median }=0 \\
95 \% C l=4.2) \\
(57.7 \%) \text { full } \\
\text { functional } \\
\text { capacity }] \\
(p=0.0001 \text { vs } \\
\text { AGA-5ARi) }\end{array}$ & $\begin{array}{l}0 \text { (Median }=0 ; \\
95 \% \mathrm{Cl}=0)[52 \\
(100 \%) \text { full } \\
\text { functional } \\
\text { capacity }] \\
(p=0.003 \text { vs no- } \\
5 \text { ARi) }\end{array}$ & 0.0002 \\
\hline $\begin{array}{l}\text { vs Day } 0 \\
(p \text {-value })\end{array}$ & 0.0001 & 0.0024 & 0.0004 & $0.98(n / s)$ & \\
\hline $\begin{array}{l}\text { Day } 7 \\
\text { (p-value) }\end{array}$ & $\begin{array}{l}1.6 \pm 6.4 \\
\text { (Median = } 0 ; \\
95 \% \mathrm{Cl}=0.7) \\
\text { (91.7\% full } \\
\text { functional } \\
\text { capacity) }\end{array}$ & $\begin{array}{l}1.1 \pm 4.9 \text { (Median } \\
=0 ; 95 \% \mathrm{Cl}=0.7) \\
{[180(93.8 \%) \text { full }} \\
\text { functional } \\
\text { capacity] } \\
(\mathrm{n} / \mathrm{s}(\mathrm{p}=0.089) \text { vs } \\
\text { AGA no-ARi) } \\
(\mathrm{n} / \mathrm{s} \text { vs AGA- } \\
5 A R i)\end{array}$ & $\begin{array}{l}4.2 \pm 10.3 \\
(\text { Median =0; } \\
95 \% C l=2.4)[57 \\
(80.3 \%) \text { full } \\
\text { functional } \\
\text { capacity] } \\
\text { (n/s vs AGA- } \\
5 A R i)\end{array}$ & $\begin{array}{l}0 \text { (Median =0; } \\
95 \% \mathrm{Cl}=0)[52 \\
(100 \%) \text { full } \\
\text { functional } \\
\text { capacity] } \\
\text { (n/s }(p=0.062) \text { vs } \\
\text { no-5ARi) }\end{array}$ & $\begin{array}{l}0.12 \\
(\mathrm{n} / \mathrm{s})\end{array}$ \\
\hline $\begin{array}{l}\text { vs Day } 0 \\
(p \text {-value })\end{array}$ & $<0.0001$ & $<0.0001$ & $<0.0001$ & $0.98(n / s)$ & \\
\hline $\begin{array}{l}\text { vs Day } 3 \\
\text { ( } p \text {-value) }\end{array}$ & 0.0037 & 0.019 & 0.016 & $1.0(\mathrm{n} / \mathrm{s})$ & \\
\hline $\begin{array}{l}\text { Day } 14 \\
\text { (p-value) }\end{array}$ & $\begin{array}{l}0.3 \pm 2.2 \\
(\text { Median }=0 ; \\
95 \% \mathrm{Cl}=0.2) \\
(98.4 \% \text { full }\end{array}$ & $\begin{array}{l}0.1 \pm 1.0(\text { Median } \\
=0 ; 95 \% \mathrm{Cl}=0.1) \\
{[190(98.6 \%) \text { full }}\end{array}$ & $\begin{array}{l}0.8 \pm 4.4 \\
(\text { Median =0; } \\
95 \% C l=1.0)[68 \\
(95.8 \%) \text { full }\end{array}$ & $\begin{array}{l}0(\text { Median }=0 ; \\
95 \% \mathrm{Cl}=0)[52 \\
(100 \%) \text { full }\end{array}$ & $\begin{array}{l}1.0 \\
(\mathrm{n} / \mathrm{s})\end{array}$ \\
\hline
\end{tabular}




\begin{tabular}{|c|c|c|c|c|c|}
\hline & $\begin{array}{l}\text { functional } \\
\text { capacity) }\end{array}$ & $\begin{array}{l}\text { functional } \\
\text { capacity] } \\
\text { (n/s vs AGA no- } \\
\text { ARi) } \\
\text { (n/s vs AGA- } \\
5 A R i)\end{array}$ & $\begin{array}{l}\text { functional } \\
\text { capacity] } \\
\text { (n/s vs AGA- } \\
5 A R i)\end{array}$ & $\begin{array}{l}\text { functional } \\
\text { capacity] } \\
(n / s(n / s \text { vs no- } \\
5 A R i)\end{array}$ & \\
\hline $\begin{array}{l}\text { vs Day } 0 \\
\text { ( } p \text {-value) }\end{array}$ & $<0.0001$ & $<0.0001$ & $<0.0001$ & $0.98(n / s)$ & \\
\hline $\begin{array}{l}\text { vs Day } 7 \\
\text { (p-value) }\end{array}$ & 0.008 & $0.54(\mathrm{n} / \mathrm{s})$ & $0.11(n / s)$ & $1.0(\mathrm{n} / \mathrm{s})$ & \\
\hline $\begin{array}{l}\text { Day } 30 \\
\text { (p-value) }\end{array}$ & $\begin{array}{l}0 \text { (Median = }=0 ; \\
95 \% \mathrm{Cl}=0) \\
(100 \% \text { full } \\
\text { functional } \\
\text { capacity })\end{array}$ & $\begin{array}{l}0 \text { (Median =0; } \\
95 \% \mathrm{Cl}=0) \text { [192 } \\
(100 \%) \text { full } \\
\text { functional } \\
\text { capacity] (n/s } \\
\text { vs AGA no-ARi) } \\
\text { (n/s vs AGA- } \\
5 A R i)\end{array}$ & $\begin{array}{l}0 \text { (Median =0; } \\
95 \% \mathrm{Cl}=0)[71 \\
(100 \%) \text { full } \\
\text { functional } \\
\text { capacity] (n/s } \\
\text { vs AGA-5ARi) }\end{array}$ & $\begin{array}{l}0 \text { (Median =0; } \\
95 \% \mathrm{Cl}=0) \text { [52 } \\
\text { (100\%) full } \\
\text { functional } \\
\text { capacity] (n/s vs } \\
\text { no-5ARi) }\end{array}$ & $\begin{array}{l}1.0 \\
(\mathrm{n} / \mathrm{s})\end{array}$ \\
\hline
\end{tabular}

$\mathrm{AGA}=$ androgenetic alopecia; $5 \mathrm{ARi}=$ Androgen receptor inhibitors; $\mathrm{n} / \mathrm{s}=$ non-significant; $\mathrm{Cl}=$ confidence interval

Table 14. Chest CT scan. 


\begin{tabular}{|c|c|c|c|c|c|}
\hline $\begin{array}{l}\text { Chest CT } \\
\text { scan (\% } \\
\text { of lungs } \\
\text { affected) }\end{array}$ & $\begin{array}{l}\text { Overall males } \\
(n=305)\end{array}$ & $\begin{array}{l}\text { Non-AGA males } \\
(n=192)\end{array}$ & $\begin{array}{l}\text { AGA no-5ARi } \\
\text { males } \\
(n=71)\end{array}$ & $\begin{array}{l}\text { AGA-5ARi } \\
(n=52)\end{array}$ & $\begin{array}{l}\text { p-value } \\
\text { (overall) }\end{array}$ \\
\hline $\begin{array}{l}\text { Day } 0 \\
\text { (p-value) }\end{array}$ & $\begin{array}{l}21.1 \pm 19.1 \\
(\text { Median = } 17.5 ; \\
95 \% \mathrm{Cl}=2.1)[30 \\
(25.9 \%) \text { normal } \\
\text { lungs of } 116 \\
\text { patients that } \\
\text { performed chest } \\
\text { CT scan in day } \\
\text { o] }\end{array}$ & $\begin{array}{l}16.0 \pm 13.9 \\
(\text { Median = } 10 ; \\
95 \% \mathrm{Cl}=2.0)^{2}[15 \\
(26.3 \%) \text { normal } \\
\text { lungs of } 57 \\
\text { patients that } \\
\text { performed chest } \\
\text { CT scan in day } \\
\text { o] } \\
(p<0.0001 \text { vs } \\
\text { AGA no-ARi) } \\
(p=0.0007 \text { vs } \\
\text { AGA-5ARi) }\end{array}$ & $\begin{array}{l}32.3 \pm 20.2 \\
(\text { Median = } 40 ; \\
95 \% \mathrm{Cl}=4.7)^{\prime}[5 \\
(10.9 \%) \text { normal } \\
\text { lungs of } 46 \\
\text { patients that } \\
\text { performed chest } \\
\text { CT scan in day } \\
\text { o] } \\
(p<0.0001 \text { vs } \\
\text { AGA-5ARi) }\end{array}$ & $\begin{array}{l}2.3 \pm 4.2 \\
(\text { Median = } 0 \text {; } \\
95 \% C l=1.1)[10 \\
(76.9 \%) \text { normal } \\
\text { lungs of } 13 \\
\text { patients that } \\
\text { performed chest } \\
C T \text { scan in day } \\
\text { o] } \\
(p<0.0001 \text { vs } \\
\text { no-5ARi) }\end{array}$ & $\begin{array}{l}< \\
0.0001\end{array}$ \\
\hline $\begin{array}{l}\text { Day } 7 \\
\text { (p-value) }\end{array}$ & $\begin{array}{l}18.8 \pm 18.9 \\
\text { (Median = 10; } \\
95 \% \mathrm{Cl}=2.1)^{2}[19 \\
(32.8 \%) \text { normal } \\
\text { lungs of } 58 \\
\text { patients that } \\
\text { performed chest } \\
\text { CT scan in day } \\
7]\end{array}$ & $\begin{array}{l}15.3 \pm 12.8 \\
(\text { Median = } 10 ; \\
95 \% C l=1.8)^{15} \\
(26.3 \%) \text { normal } \\
\text { lungs of } 19 \\
\text { patients that } \\
\text { performed chest } \\
C T \text { scan in day } \\
7] \\
(p=0.02 \text { vs } A G A \\
\text { no-ARi) } \\
(p=0.0015 \text { vs } \\
\text { AGA-5ARi) }\end{array}$ & $\begin{array}{l}29.3 \pm 19.8 \\
(\text { Median = } 25 ; \\
95 \% C l=4.6)[3 \\
(11.1 \%) \text { normal } \\
\text { lungs of } 27 \\
\text { patients that } \\
\text { performed chest } \\
\text { CT scan in day } \\
7] \\
(p<0.0001 \text { vs } \\
\text { AGA-5ARi) }\end{array}$ & $\begin{array}{l}0.8 \pm 2.8 \\
(\text { Median =0; } \\
95 \% C l=0.7)[11 \\
(91.7 \%) \text { normal } \\
\text { lungs of } 12 \\
\text { patients that } \\
\text { performed chest } \\
\text { CT scan in day } \\
7] \\
(p<0.0001 \text { vs } \\
\text { no-5ARi) }\end{array}$ & $\begin{array}{l}< \\
0.0001\end{array}$ \\
\hline $\begin{array}{l}\text { vs Day } 0 \\
(p \text {-value })\end{array}$ & $0.32(n / s)$ & $0.091(n / s)$ & $0.62(n / s)$ & $1.0(n / s)$ & \\
\hline $\begin{array}{l}\text { Day } 14 \\
\text { (p-value) }\end{array}$ & $\begin{array}{l}18.6 \pm 16.0 \\
\text { (Median = 10; } \\
95 \% \mathrm{Cl}=1.8) \text { ' } 16 \\
(24.6 \%) \text { normal } \\
\text { lungs of } 65 \\
\text { patients that } \\
\text { performed chest } \\
\text { CT scan in day } \\
14]\end{array}$ & $\begin{array}{l}11.1 \pm 11.2 \\
\text { (Median = } 10 ; \\
95 \% \mathrm{Cl}=1.6) \text { [14 } \\
\text { (38.9\%) normal } \\
\text { lungs of } 36 \\
\text { patients that } \\
\text { performed chest } \\
\text { CT scan in day } \\
14] \\
\text { (p<0.0001 vs } \\
\text { AGA no-ARi) } \\
\text { (n/a vs AGA- } \\
5 \text { ARi) }\end{array}$ & $\begin{array}{l}28.6 \pm 16.2 \\
(\text { Median = } 25 ; \\
95 \% C l=3.8)[2 \\
(7.1 \%) \text { normal } \\
\text { lungs of } 28 \\
\text { patients that } \\
\text { performed chest } \\
\text { CT scan in day } \\
14] \\
(n / a)\end{array}$ & $\begin{array}{l}10 \text { (01 patient } \\
\text { that performed } \\
\text { chest CT scan } \\
\text { in day } 14) \\
(n / a)\end{array}$ & $\mathrm{n} / \mathrm{a}$ \\
\hline vs Day 0 & $0.15(n / s)$ & $0.12(\mathrm{n} / \mathrm{s})$ & $0.45(n / s)$ & $1.0(n / s)$ & \\
\hline
\end{tabular}




\begin{tabular}{|c|c|c|c|c|}
\hline $\begin{array}{l}\text { vs Day } 7 \\
\text { (p-value) }\end{array}$ & $0.21(n / s)$ & $0.28(n / s)$ & $0.85(n / s)$ & $1.0(n / s)$ \\
\hline $\begin{array}{l}\text { Day } 30 \\
\text { (p-value) }\end{array}$ & $\begin{array}{l}19.1 \pm 13.0 \\
\text { (Median = 17.5; } \\
95 \% \mathrm{Cl}=1.4)[4 \\
\text { (12.5\%) normal } \\
\text { lungs of } 32 \\
\text { patients that } \\
\text { performed chest } \\
\text { CT scan in day } \\
\text { 30] }\end{array}$ & $\begin{array}{l}10.0 \pm 7.9 \\
(\text { Median }=10 ; \\
95 \% \mathrm{Cl}=1.1)^{[}[3 \\
(25.0 \%) \text { normal } \\
\text { lungs of } 12 \\
\text { patients that } \\
\text { performed chest } \\
C T \text { scan in day } \\
30] \\
(p=0.004 \text { vs } A G A \\
\text { no-ARi) } \\
\text { (n/a vs AGA- } \\
5 \text { ARi) }\end{array}$ & $\begin{array}{l}24.5 \pm 12.4 \\
(\text { Median }=25 ; \\
95 \% \mathrm{Cl}=2.9)^{\prime}[1 \\
(5.0 \%) \text { normal } \\
\text { lungs of } 20 \\
\text { patients that } \\
\text { performed chest } \\
\text { CT scan in day } \\
30] \\
(\mathrm{n} / \mathrm{a})\end{array}$ & $\begin{array}{l}\text { No patients } \\
\text { performed chest } \\
\text { CT scan in day } \\
30 \\
(n / a)\end{array}$ \\
\hline $\begin{array}{l}\text { vs Day } 0 \\
\text { ( } p \text {-value })\end{array}$ & $0.41(n / s)$ & $0.27(n / s)$ & $0.13(n / s)$ & $1.0(n / s)$ \\
\hline $\begin{array}{l}\text { vs Day } 7 \\
\text { (p-value) }\end{array}$ & $0.44(n / s)$ & $0.36(n / s)$ & $0.51(\mathrm{n} / \mathrm{s})$ & $1.0(n / s)$ \\
\hline $\begin{array}{l}\text { vs Day } \\
14 \\
\text { (p-value) }\end{array}$ & $0.70(n / s)$ & $0.97(n / s)$ & $0.38(n / s)$ & $1.0(n / s)$ \\
\hline
\end{tabular}

Table 15. COVID-19 progression outcomes. 


\begin{tabular}{|c|c|c|c|c|c|}
\hline Disease progression outcomes & $\begin{array}{l}\text { Overall } \\
\text { males } \\
(n=305)\end{array}$ & $\begin{array}{l}\text { Non-AGA } \\
\text { males } \\
(n=192)\end{array}$ & $\begin{array}{l}\text { AGA no-5ARi } \\
\text { males } \\
(n=71)\end{array}$ & $\begin{array}{l}\text { AGA- } \\
5 \text { ARi } \\
(n= \\
52)\end{array}$ & $\begin{array}{l}\text { p-value } \\
\text { (overall) }\end{array}$ \\
\hline $\begin{array}{l}\text { Brescia COVID-19 Respiratory } \\
\text { Severity Scale }(0-4)\end{array}$ & 0 & 0 & 0 & 0 & $\begin{array}{l}1.00 \\
(\mathrm{n} / \mathrm{s})\end{array}$ \\
\hline Hospitalization & 0 & 0 & 0 & 0 & $\begin{array}{l}1.00 \\
(\mathrm{n} / \mathrm{s})\end{array}$ \\
\hline Mechanical ventilation & 0 & 0 & 0 & 0 & $\begin{array}{l}1.00 \\
(\mathrm{n} / \mathrm{s})\end{array}$ \\
\hline Noradrenaline/dopamine & 0 & 0 & 0 & 0 & $\begin{array}{l}1.00 \\
(\mathrm{n} / \mathrm{s})\end{array}$ \\
\hline Death & 0 & 0 & 0 & 0 & $\begin{array}{l}1.00 \\
(\mathrm{n} / \mathrm{s})\end{array}$ \\
\hline
\end{tabular}

\section{Discussion}

The importance of clinical characterization during early COVID-19

To our knowledge, this is the first thorough characterization of COVID-19 in its earlier stages. The improvement on the knowledge regarding COVID-19 clinical characteristics and course is essential for earlier detection and more successful pharmacological antiviral approaches, that can eventually lead to decreased need for hospitalization, mechanical ventilation, and other complications.

The large heterogeneity of clinical manifestations among infected patients and the insistent requirement for the presence of fever as a sign of suspected COVID-19 are probably the major reasons for the persistent lack of an accurate characterization of how patients behave clinically during the first stage of COVID-19 (11-13).

Indeed, when actively questioned, unspecific symptoms that patients tend to underestimate are actually key signs for an early diagnosis of COVID-19. During the present prospective study, four clinical clusters of COVID-19 manifestations have been identified: anosmia-Ageusia dominance, dengue-like, URTI-like, and GI infection-like clinical presentation, besides those cases that mix two or more of these clusters, or only present unspecific symptoms, such as fever or headache.

Baseline characteristics, diseases that could lead to worse outcomes in COVID-19 and use of drug classes that could attenuate or aggravate COVID-19 presentation were similar between groups, which hampers from population selection bias for the present analysis. 
The slightly lower choice for hydroxychloroquine in the AGA group may be due to the apparent higher prevalence of cardiovascular and metabolic diseases in this group, as a clinical attempt to avoid cardiovascular adverse effects from its use. Since all other drugs, including the additional ones, were used at a similar extent between groups, differences in terms of COVID-19 progression cannot be justified by these differences.

In terms of therapies prescribed as additional approaches aiming to provide a more comprehensive protection, Xa factor inhibitors tended to be given to intermediate whereas enoxaparin was given to patients at high risk of developing thrombosis, although these anticoagulants were prescribed at a subjective, clinical judgement basis. Since time-to-treat was higher among higher-risk patients, partially due to the slower progression and longer period until appearance of anosmia or ageusia, patients under enoxaparin were coincidently prescribed with glucocorticoids. Although both anticoagulants and glucocorticoids could decrease disease progression and duration, they were insufficient to equalize these characteristics to patients at lower risk. However, although uncertain, it is possible that the use of these drug classes before seven days of disease may have contributed to the lack of progression to hospitalization and other more severe outcomes.

A thorough description of the patients' medical history and a complete characterization of the COVID-19 presentation are critical for an accurate analysis of the predictions, and to provide a more appropriate and accurate therapeutic approach.

COVID-19 course and severity

Overall, in regards with clinical manifestations, mean time to appearance of symptoms were less different compared to duration.

Disease duration was notably higher in AGA males not treated with dutasteride, in accordance with literature $(5,6,14,16,17)$, whereas the chronic use of dutasteride likely prevented the appearance of symptoms in almost all patients, possibly due to its indirect inhibitory effects on the expression of TMPRSS-2, a protein that facilitates the SARS-CoV-2 entry in the cells. This is in full correspondence with recent literature on dutasteride ability to diminish severe COVID-19 in hospitalized patients (18). Whether this is applicable for finasteride, another drug of the same class as dutasteride, is uncertain, although unlikely, due to the narrower actions of finasteride, compared to dutasteride.

If one considers that dutasteride exerted an important role in COVID-19 attenuation, the larger use of dutasteride in AGA no-5ARi patients compared to non-AGA when COVID-19 was diagnosed could have attenuated the differences in disease severity and duration between these groups. Although differences between non-AGA and AGA no-5ARicould have been more pronounced in case dutasteride had not been used, differences still remained, which demonstrates that benefits provided by dutasteride are at least partially due to its chronic and more structural effects. 
Although it is unlikely that there were differences between hydroxychloroquine, nitazoxanide or ivermectin, these treatments will be compared in a specific head-to-head comparison analysis. Unlike dutasteride, spironolactone seems to have provided additional benefits, even when prescribed after COVID-19 diagnosis, at least when diagnosed early, which meets corresponding rationale and clinical observations (19-21), although specific analyses and an RCT is currently ongoing to confirm this finding.

Clinical recovery speed became noticeably quicker after Day 1 of treatment, regardless of the time-to-treat and drug combination offered, which reinforces the hypothesis on the efficacy of the use of early treatments for COVID-19.

Compared to other groups, AGA no-5ARi had slower clinical course, although not milder progression of symptoms until diagnosis of COVID-19, which may justify why this group was more affected since Days -7 to -4 .

While disease duration was shorter in non-AGA, the speed of clinical recovery was similar between AGA no-5ARi and non-AGA, probably because AGA males experimented a longer period of clinical manifestations until treatment began (longer time-to-treat). When summed, the pre- and post-treatment initiation periods were longer in AGA no-5ARi males.

Although time-to-treat was apparently correlated with duration of manifestations in a linear manner, at least among our patients, any delay in the initiation of one of the proposed anti-COVID therapies did not affect the ability to prevent hospitalization.

The lack of correlation between clinical and radiological improvements may be due to the extensively described persistence of the imaging of compromised lungs for several weeks after COVID-19.

The current prevailing criteria used for COVID-19 severity, including WHO COVID Scale and Brescia Respiratory Scale, were found to be inappropriate as being the only scales to be employed for studies in patients in early COVID-19.

\section{Comparison with current literature}

Overall, when compared to current literature on COVID-19 clinical characterization of COVID-19, the percentages of each symptom described in the present study were similar. However, unusual or less noticed symptoms, including diarrhea and myalgia, that only recently have been reported as possible presentations of COVID-19, were present in a substantial number of patients (24-27).

The rate and speed of recovery were overwhelmingly better compared to untreated patients with similar characteristics. Indeed, our previous interim analysis of the present prospective observational study demonstrated the undisputable differences $(11-13 ; 24 ; 25)$, which precluded us from performing a full placebo-control study in our RCT.

Final discussion

Page 52/57 
This is a comprehensive prospective observational study that evaluated different aspects of COVID-19 in males, as well as its clinical course when diagnosed and treated early.

AGA males presented larger number, more severe, and longer symptom duration, and prolonged period of time until COVID-19 clinical and laboratorial remission, compared to non-AGA males. However, the chronic use of dutasteride seemed not only seemed to completely mitigate the additional risk of AGA in males, but also to decrease COVID-19 severity to levels substantially lower than non-AGA males.

The early use of medications proposed as being effective for COVID-19 due to their direct or indirect antiviral therapy, at least in patients diagnosed during the first stage of the disease, has demonstrated indisputable improved COVID-19 related clinical outcomes compared to the extensively described COVID19 clinical course, and avoided the progression to more severe states, including hospitalization and mechanical ventilation, in all patients included in the present analysis. The dramatic improvement occurred in an independent manner from all major factors of higher risk for COVID-19 complications, demonstrating that obesity, comorbidities, aging and AGA as risk factors can be completely mitigated by the combination of more sensitive clinical suspect with early pharmacological approaches.

Whether the earlier diagnosis of COVID-19 based on a more sensitive case-detection basis had at least a partial role in the improved outcomes compared to the literature is uncertain, although possible.

The overwhelming differences indicate that full placebo control RCTs for early COVID-19 may be ethically questionable. Instead, double blind therapies with different options, or mixed open label placebo control for COVID-19 should be considered.

\section{Conclusion}

Males with androgenetic alopecia (AGA) presented more pronounced COVID-19 compared to non-AGA males, which was fully mitigated by chronic dutasteride use, that yielded fewer symptoms, even when compared to non-AGA males.

Drug combinations between azithromycin and hydroxychloroquine, nitazoxanide, or ivermectin, for patients recently diagnosed during the first stage of COVID-19, had noticeable improved clinical outcomes and complete remission of hospitalization, at least in the population of the present study, and completely mitigated additional risks due to obesity, aging, presence of comorbidities, or AGA.

\section{Declarations}

Funding statements

The funding of present study was fully supported by Corpometria Institute (Brasilia, DF, Brazil) and Applied Biology Inc (Irvine, CA, USA).

Conflict of interest statement 
Authors declare no conflict of interest with any of the pharmacological interventions proposed by the present study..

\section{References}

1. Guan W, Ni Z, Hu Y, et al. Clinical characteristics of coronavirus disease 2019 in China. N Engl J Med 2020; Feb 28.

2. Hajifathalian K, Kumar S, Newberry C, et al. Obesity is associated with worse outcomes in COVID-19: Analysis of Early Data From New York City [published online ahead of print, 2020 May 29]. Obesity (Silver Spring). 2020;10.1002/oby.22923.

3. Kalligeros M, Shehadeh F, Mylona EK, et al. Association of Obesity with Disease Severity among Patients with COVID-19 [published online ahead of print, 2020 Apr 30]. Obesity (Silver Spring). 2020;10.1002/oby.22859.

4. Palaiodimos L, Kokkinidis DG, Li W, et al. Severe obesity, increasing age and male sex are independently associated with worse in-hospital outcomes, and higher in-hospital mortality, in a cohort of patients with COVID-19 in the Bronx, New York [published online ahead of print, 2020 May 16]. Metabolism. 2020;108:154262.

5. Goren A, Vano-Galvan S, Wambier CG, et al. A preliminary observation: male pattern hair loss among hospitalized COVID-19 patients in Spain - A potential clue to the role of androgens in COVID-19 severity [published online ahead of print, 2020 Apr 16].J Cosmet Dermatol. 2020;10.1111/jocd.13443.

6. Goren A, McCoy J, Wambier CG, et al. What does androgenetic alopecia have to do with COVID-19? An insight into a potential new therapy [published online ahead of print, $2020 \mathrm{Apr}$ 1].Dermatol Ther. 2020;e13365.

7. Zhou F, Yu T, Du R, et al. Clinical course and risk factors for mortality of adult inpatients with COVID19 in Wuhan, China: a retrospective cohort study [published online ahead of print, 2020 Mar 11] [published correction appears in Lancet. 2020 Mar 12;:]. Lancet. 2020;S0140-6736(20)30566-3.

8. Lauer SA, Grantz KH, Bi Q, et al. The Incubation Period of Coronavirus Disease 2019 (COVID-19) From Publicly Reported Confirmed Cases: Estimation and Application [published online ahead of print, 2020 Mar 10]. Ann Intern Med. 2020;10.7326/M20-0504.

9. Wu C, Chen X, Cai Y, et al. Risk Factors Associated With Acute Respiratory Distress Syndrome and Death in Patients With Coronavirus Disease 2019 Pneumonia in Wuhan, China [published online ahead of print, 2020 Mar 13]. JAMA Intern Med. 2020;10.1001/jamainternmed.2020.0994.

10. Wu C, Chen X, Cai Y, et al. Risk Factors Associated With Acute Respiratory Distress Syndrome and Death in Patients With Coronavirus Disease 2019 Pneumonia in Wuhan, China [published online ahead of print, 2020 Mar 13]. JAMA Intern Med. 2020;10.1001/jamainternmed.2020.0994.

11. https://www.who.int/teams/health-care-readiness-clinical-unit/covid-19/data-platform (Last accessed September 29th, 2020) 
12. https://www.cdc.gov/coronavirus/2019-ncov/hcp/clinical-guidance-management-patients.html (Last accessed September 29th, 2020)

13. Stokes EK, Zambrano LD, Anderson KN, et al. Coronavirus Disease 2019 Case Surveillance - United States, January 22-May 30, 2020. MMWR Morb Mortal Wkly Rep 2020; 69:759.

14. Cadegiani, F.A. Repurposing existing drugs for COVID-19: an endocrinology perspective.BMC Endocr Disord 20, 149 (2020). https://doi.org/10.1186/s12902-020-00626-0

15. Pascarella G, Strumia A, Piliego C, Bruno F, Del Buono R, Costa F, Scarlata S, Agrò FE. COVID-19 diagnosis and management: a comprehensive review. J Intern Med. 2020 Aug;288(2):192-206. doi: 10.1111/joim.13091.

16. McCoy J, Wambier CG, Herrera S, Vaño-Galván S, Gioia F, Comeche B, Ron R, Serrano-Villar S, Iwasiow RM, Tayeb MA, Cadegiani FA, Mesinkovska NA, Shapiro J, Sinclair R, Goren A. Androgen Receptor Genetic Variant Predicts COVID-19 Disease Severity: A Prospective Longitudinal Study of Hospitalized COVID-19 Male Patients. J Eur Acad Dermatol Venereol. 2020 Sep 25.

17. Kragholm K, Andersen MP, Gerds TA, et al. Association between male sex and outcomes of Coronavirus Disease 2019 (Covid-19) - a Danish nationwide, register-based study. Clin Infect Dis 2020.

18. Goren A, Wambier CG, Herrera S, McCoy J, Vaño-Galván S, Gioia F, Comeche B, Ron R, Serrano-Villar S, Ramos PM, Cadegiani FA, Kovacevic M, Tosti A, Shapiro J, Sinclair R. Anti-androgens may protect against severe COVID-19 outcomes: results from a prospective cohort study of 77 hospitalized men. J Eur Acad Dermatol Venereol. 2020 Sep 25. doi: 10.1111/jdv.16953.

19. Cadegiani FA. Can spironolactone be used to prevent COVID-19-induced acute respiratory distress syndrome in patients with hypertension? Am J Physiol Endocrinol Metab. 2020 May 1;318(5):E587E588. doi: 10.1152/ajpendo.00136.2020.

20. Cadegiani FA, Wambier CG, Goren A. Spironolactone: An Anti-androgenic and Anti-hypertensive Drug That May Provide Protection Against the Novel Coronavirus (SARS-CoV-2) Induced Acute Respiratory Distress Syndrome (ARDS) in COVID-19. Front Med (Lausanne). 2020 Jul 28;7:453. doi: 10.3389/fmed.2020.00453.

21. Cadegiani FA, Goren A, Wambier CG. Spironolactone may provide protection from SARS-CoV-2: Targeting androgens, angiotensin converting enzyme 2 (ACE2), and renin-angiotensin-aldosterone system (RAAS). Med Hypotheses. 2020 Jul 16;143:110112. doi: 10.1016/j.mehy.2020.110112.

22. Machin D, Campbell MJ, Tan SB, Tan SH (2009) Sample size tables for clinical studies. $3^{\text {rd }}$ Chichester: Wiley-Blackwell.

23. Richardson S, Hirsch JS, Narasimhan M, Crawford JM, McGinn T, Davidson KW; the Northwell COVID19 Research Consortium, Barnaby DP, Becker LB, Chelico JD, Cohen SL, Cookingham J, Coppa K, Diefenbach MA, Dominello AJ, Duer-Hefele J, Falzon L, Gitlin J, Hajizadeh N, Harvin TG, Hirschwerk DA, Kim EJ, Kozel ZM, Marrast LM, Mogavero JN, Osorio GA, Qiu M, Zanos TP. Presenting Characteristics, Comorbidities, and Outcomes Among 5700 Patients Hospitalized With COVID-19 in 
the New York City Area. 2020 May 26;323(20):2052-2059. doi: 10.1001/jama.2020.6775. Erratum in: JAMA. 2020 May 26;323(20):2098.

24. https://www.uptodate.com/contents/coronavirus-disease-2019-covid-19-clinical-features? topicRef $=126981 \&$ source $=$ see_link (Last accessed September 29th, 2020)

25. Kim GU, Kim MJ, Ra SH, Lee J, Bae S, Jung J, Kim SH. Clinical characteristics of asymptomatic and symptomatic patients with mild COVID-19. Clin Microbiol Infect. 2020 Jul;26(7):948.e1-948.e3. doi: 10.1016/j.cmi.2020.04.040.

26. Lechien JR, Chiesa-Estomba CM, De Siati DR et al. Olfactory and gustatory dysfunctions as a clinical presentation of mild-to-moderate forms of the coronavirus disease (COVID-19): a multicenter European study. Eur Arch Otorhinolaryngol. 2020 Aug;277(8):2251-2261. doi: 10.1007/s00405-02005965-1.

27. Tong JY, Wong A, Zhu D, Fastenberg JH, Tham T. The Prevalence of Olfactory and Gustatory Dysfunction in COVID-19 Patients: A Systematic Review and Meta-analysis. Otolaryngol Head Neck Surg. 2020 Jul;163(1):3-11. doi: 10.1177/0194599820926473.

\section{Figures}

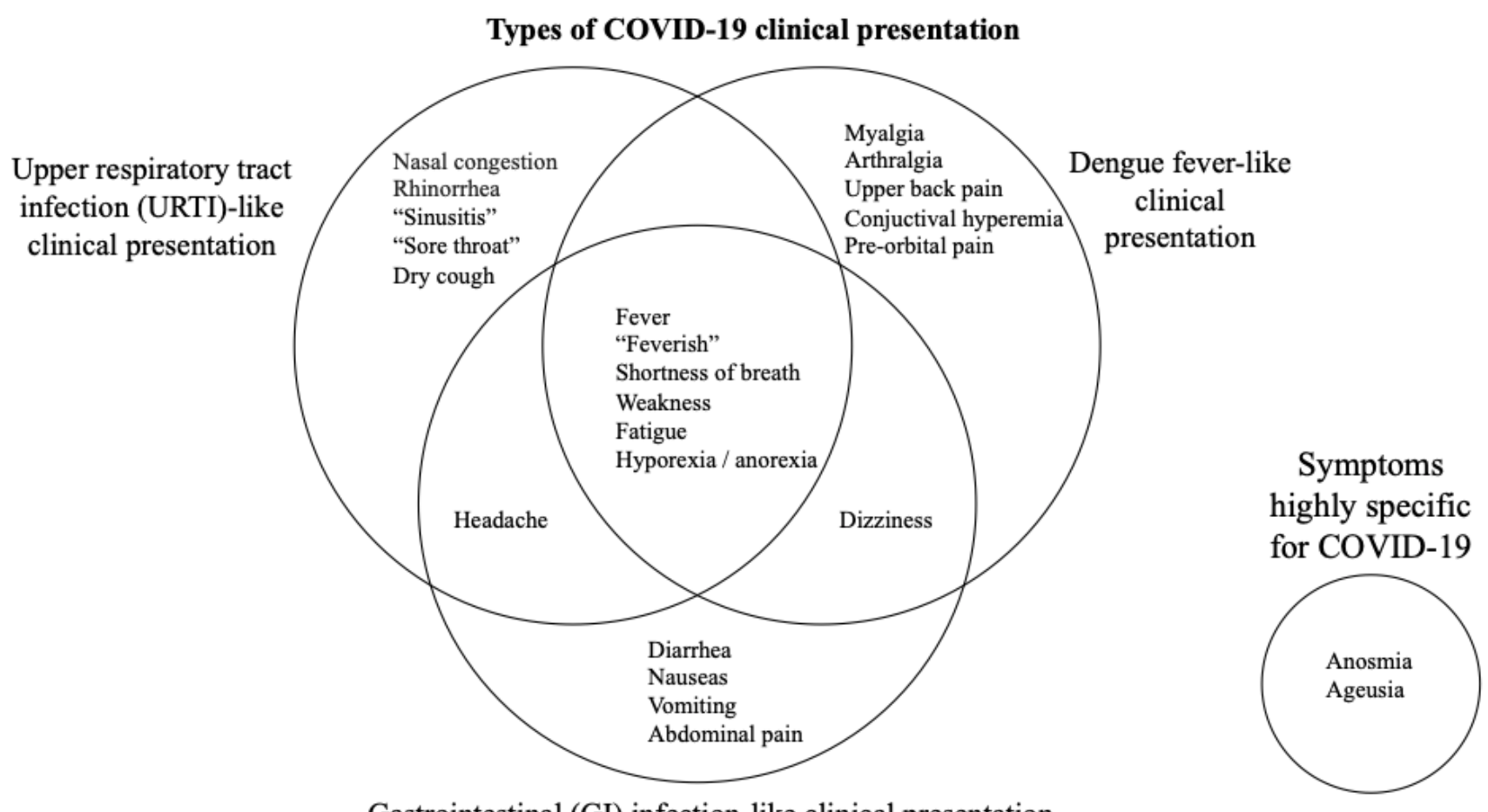

Figure 1 
Clusters of COVID-19 clinical presentation 